\title{
CaWRKY28 Cys249 is Required for Interaction with CaWRKY40 in the Regulation of Pepper Immunity to Ralstonia solanacearum
}

\author{
Sheng Yang, ${ }^{1,2,3}$ Yangwen Zhang, ${ }^{1,2,3}$ Weiwei Cai, ${ }^{1,2,3}$ Cailing Liu, ${ }^{1,2,3}$ Jiong Hu, ${ }^{1,2,3}$ Lei Shen, ${ }^{1,2,3}$ \\ Xueying Huang, ${ }^{1,2,3}$ Deyi Guan, ${ }^{1,2,3}$ and Shuilin $\mathrm{He}^{1,2,3, \dagger}$ \\ ${ }^{1}$ National Education Ministry Key Laboratory of Plant Genetic Improvement and Comprehensive Utilization, Fujian \\ Agriculture and Forestry University, Fuzhou, Fujian 350002, PR China \\ ${ }^{2}$ Key Laboratory of Applied Genetics of universities in Fujian Province, Fujian Agriculture and Forestry University, Fuzhou, \\ Fujian 350002, China \\ ${ }^{3}$ Agricultural College, Fujian Agriculture and Forestry University, Fuzhou, Fujian 350002, PR China
}

Accepted 6 February 2021.

\begin{abstract}
WRKY transcription factors have been implicated in plant response to pathogens but how WRKY-mediated networks are organized and operate to produce appropriate transcriptional outputs remains largely unclear. Here, we identify a member of the WRKY family from pepper (Capsicum annuum), CaWRKY28, that physically interacts with CaWRKY40, a positive regulator of pepper immunity and thermotolerance. We confirmed CaWRKY28-CaWRKY40 interaction by coimmunoprecipitation, bimolecular fluorescence complementation, and microscale thermophoresis. Our findings supported the idea that CaWRKY28 is a nuclear protein that acts as positive regulator in pepper responses to infection by the pathogenic bacterium Ralstonia solanacearum. It performs its function not by directly modulating the $\mathrm{W}$-box containing immunity-related genes but by promoting CaWRKY40 via physical interaction to bind and activate its immunity-related target genes, including CaPR1, CaNPR1, CaDEF1, and CaABR1, but not its thermotolerance-related target gene, CaHSP24. All of these data indicate that CaWRKY28 interacts with and potentiates CaWRKY40 in regulating immunity against $R$. solanacearum infection but not thermotolerance. Importantly, we discovered that CaWRKY28 Cys249, shared by CaWRKY28 and its orthologs probably only in the family Solanaceae, is crucial for the CaWRKY28-CaWRKY40 interaction. These results highlight how CaWRKY28 associates with CaWRKY40 during the establishment of WRKY networks, and how CaWRKY40
\end{abstract}

${ }^{\dagger}$ Corresponding: S. He; shlhe@fafu.edu.cn

Funding: This work was supported by grants from the National Natural Science Foundation of China (31572136, 31372061, and 31902032), the Development Fund Project of Fujian Agriculture and Forestry University (CXZX2016158 and CXZX2017548), and the Scientific Research Foundation of Graduate School of Fujian Agriculture and Forestry University (324-1122yb047).

*The $\boldsymbol{e}$-Xtra logo stands for "electronic extra" and indicates that a supplementary table and supplementary figures are published online.

The author(s) declare no conflict of interest.

(c) (1) () $\odot$ Copyright ( 92021 The Author(s). This is an open access article (c) achieves its functional specificity during pepper responses to R. solanacearum infection.

Keywords: Capsicum annuum, CaWRKY28, CaWRKY40, defense signaling pathways, pathogens, Ralstonia solanacearum, RSI, transpiration

Numerous studies have demonstrated that the plant immune response against pathogens is regulated largely at the transcriptional level and relies on the action of various transcription factors (TFs). By targeting specific promoters and regulating the underlying loci, TFs activate a massive transcriptional reprogramming leading to an effective immune response. To generate appropriate immune outputs, TFs form highly interconnected transcriptional networks (Birkenbihl et al. 2017b; Tsuda and Somssich 2015). However, how these networks are organized and operate in response to different pathogens remains poorly understood.

WRKY TFs constitute one of the largest families of plant TFs, and have been intensively studied over the past two decades (Amorim et al. 2017; Birkenbihl et al. 2017b; Pandey and Somssich 2009). Members of this family contain one or two WRKY domains, consisting of the highly conserved motif WRKYGQK at their $\mathrm{N}$ terminus, together with a novel zinc-finger-like motif (Eulgem et al. 2000; Zhang and Wang 2005). WRKY TFs bind a highly conserved cis-regulatory element, W-box (TTGACC/T), in the promoters of their target genes, thereby regulating a wide array of biological processes including growth, development, and defense response to environmental stresses. The functional specificities of WRKY TFs are determined, on the one hand, by their structures-indeed, variations in the conserved WRKY domains (such as WRKYGEK and WRKYGKK) or in the zincfinger-like motifs (Eulgem et al. 2000; Zhang and Wang 2005) - and, on the other hand, by the structure of DNA motifs (Kanofsky et al. 2017; Liu et al. 2015; Machens et al. 2014) or their adjacent bases (Ciolkowski et al. 2008). In addition, the specific targeting and, thus, the functions of WRKY TFs may also be modulated by other proteins, including WRKY TFs, via protein-protein interaction (Alves et al. 2014; Chi et al. 2013). Collectively, the above factors all contribute to the complex regulation of the functional specificity associated with a given WRKY TF, which remains poorly understood. 
One of the most essential roles of WRKY TFs is the regulation of plant defense in response to pathogen infection. Most genes encoding WRKY TFs identified in the genome of a given plant species are induced at the transcriptional level by the attack of a single pathogen (Dong et al. 2003; Pandey and Somssich 2009; Segonzac et al. 2017). These WRKY TFs are believed to assemble WRKY networks in defense signaling (Eulgem and Somssich 2007), although only a few cases have been described to date (Abeysinghe et al. 2019; Liu et al. 2012; $\mathrm{Xu}$ et al. 2006). Relying on a complex network of proteins rather than a single linear pathway may grant the plant immune system a greater resistance against suppression by effectors. However, several WRKY TFs may be protected in some host plants by quickly evolving resistance proteins (Le Roux et al. 2015; Sarris et al. 2015). How these WRKY networks are organized and operate is currently largely unclear.

The Solanaceae family comprises 3,000 to 4,000 species classified into approximately 90 genera, including economically important crops such as tobacco (Nicotiana tabacum), tomato (Solanum lycopersicum), and pepper (Capsicum annuum) (Gebhardt 2016). These crop species are very often exposed to, and infected by, soilborne pathogens such as the bacterium Ralstonia solanacearum, which causes plant wilt resulting in substantial production loss (Lebeau et al. 2011; Mamphogoro et al. 2020; Thera et al. 2010). Despite differences in the evolution of their chromosomes, genomes ( $\mathrm{Wu}$ and Tanksley 2010), and transcriptomes (Rensink et al. 2005), most genes exhibit a high degree of sequence conservation within the Solanaceae family (Rensink et al. 2005). We previously determined that a subset of WRKY TFs was induced in pepper plants in response to $R$. solanacearum infection in a deep sequencing of the transcriptome (RNA-sequencing) dataset (data in the China National GeneBank). In particular, the pepper $W R K Y$ family members CaWRKY6, CaWRKY22, CaWRKY27, CaWRKY40, CaWR$K Y 40 b$, and $C a W R K Y 58$ are involved in the immune response of pepper plants to $R$. solanacearum, during which they act as positive or negative regulators (Cai et al. 2015; Dang et al. 2013; Hussain et al. 2018; Ifnan Khan et al. 2018; Wang et al. 2013). CaWRKY40, a positive regulator in pepper response to $R$. solanacearum infection (RSI) that is targeted and transcriptionally regulated by CaWRKY6, CabZIP63, and CaWRKY40b, positively regulates $C a W R K Y 22$ and $C a W R K Y 27$ and forms positive feedback loops with CaCDPK15 and CabZIP63 (Shen et al. 2016a, b). In addition, CaWRKY40 exhibits an autoregulation capability by targeting the double-W box, which is highly conserved in the promoter of CaWRKY40 and its orthologs in other plant species, during response to pathogen attack (Liu et al. 2020). All of these results highlight a role of CaWRKY40 as central node in the WRKY TF networks and in the regulation of transcriptional reprogramming in pepper immunity against $R$. solanacearum infection. Interestingly, the majority of these WRKY TFs also participate in pepper responses to high-temperature and high-humidity stress (HTHH) (Cai et al. 2015; Dang et al. 2013; Hussain et al. 2018; Ifnan Khan et al. 2018; Wang et al. 2013), suggesting a close link between pepper resistance to $R$. solanacearum infection and $\mathrm{HTHH}$. We speculate that WRKYs may interact with other proteins to achieve their specific functions in distinct contexts. In the present study, we characterized a set of proteins that interact with pepper CaWRKY40, which acts as a critical node in the WRKY network involved in responses to both $R$. solanacearum infection and thermotolerance under high humidity (Dang et al. 2013). These putative interaction partners were isolated by pull-down assays, followed by liquid chromatography with tandem mass spectrometry (LC-MS/ MS). The other pepper WRKY TF, CaWRKY28, is one such CaWRKY40 interactor and is the focus of the present study. We further reveal that Cys249 in CaWRKY28 is critical for its interaction with and activation of CaWRKY40 and, specifically, for the regulation of pepper immunity against $R$. solanacearum.

\section{RESULTS}

CaWRKY28 interacts with CaWRKY40 in pepper plants challenged by $R$. solanacearum infection.

CaWRKY40 is a positive regulator of pepper responses to $R$. solanacearum infection as well as to thermotolerance in high humidity conditions (Dang et al. 2013). Because the molecular underpinnings of plant immunity and thermotolerance are likely to be different, we speculated that CaWRKY40 function might be modulated by specific interaction partners in order to achieve its role in response to $R$. solanacearum infection. We undertook a pull-down approach to identify CaWRKY40 interactors; fusion protein CaWRKY40-glutathione-S-transferase (GST) was expressed from Escherichia coli BL21 (DE3), which was mixed with the proteins isolated from $R$. solanacearum-inoculated pepper leaves. The mixed proteins were purified using BeaverBeads GSH and subjected to sodium dodecyl sulfate polyacrylamide gel electrophoresis (SDS-PAGE) to separate and examine the possible interacting proteins of WRKY40. The possible interactors were cut off for the LC-MS/MS assay (Pennington et al. 2016). Among candidate interacting proteins, we identified a putative WRKY protein, whose deduced amino acid sequence (GenBank ID: KAF3622752.1) exhibited the highest similarity to WRKY28 in Arabidopsis thaliana. Therefore, we designated the encoding gene CaWRKY28 (renamed from WRKY23 from the pepper genome project). CaWRKY28 also showed high sequence similarity with putative orthologs in other Solanaceae species, including NtWRKY71 in tobacco, SIWRKY71 in tomato, StWRKY71 in potato (S. tuberosum), and NsWRKY71 in N. sylvestris. On the basis of the presence of a single and highly conserved WRKY domain and zinc-finger domain (C-X4-C-X23-H-X1-H), CaWRKY28 is a member of the group II WRKY proteins in pepper. However, the presence of a conserved Cys249 residue in CaWRKY28 and its Solanaceae orthologs, but not in other plant species such as AtWRKY28 or OsWRKY28, also gives CaWRKY28 and Solanaceae orthologs attributes of group III WRKY members (Supplementary Fig. S1).

\section{Confirmation of the CaWRKY28-CaWRKY40 interaction.}

To confirm the interaction between CaWRKY28 and CaWRKY40, we first performed a coimmunoprecipitation (Co-IP) assay. For the Co-IP assay, the total protein was isolated from pepper leaves coinfiltrated with Agrobacterium tumefaciens strain GV3101 cells containing 35S:CaWRKY28-FLAG and 35S:CaWRKRY40-HA (at a $1: 1$ ratio) at $48 \mathrm{~h}$ postinfiltration (hpi), and was incubated with antibody of HA to immunoprecipitate CaWRKRY40-HA. The presence of CaWRKY28-FLAG in copurified proteins was tested by immunoblotting with an antiFLAG antibody. This analysis confirmed that CaWRKY28 interacts with CaWRKY40 (Fig. 1A). In addition, we determined whether CaWRKY28 and CaWRKY40 interacted in planta by a bimolecular fluorescence complementation (BiFC) assay, for which CaWRKY28 was fused to the $\mathrm{N}$ terminus of the yellow fluorescent protein (YFP) $\left(\right.$ CaWRKY28-YFP $\left.{ }^{N}\right)$, while CaWRKY40 was fused to the C terminus of YFP (CaWRKY40$\left.Y F P^{C}\right)$. We also generated the reciprocal set of constructs $\left(C a W R K Y 28-Y F P^{C}\right.$ and CaWRKY40-YFP ${ }^{N}$ ). We tested for the interaction between the fusion proteins in $N$. benthamiana leaves via Agrobacterium-mediated transient expression at 48 hpi. Indeed, we detected YFP fluorescence in plants coexpressing both CaWRKY28 and CaWRKY40 fusion proteins but not in control plants. In addition, we observed YFP fluorescence only in the nucleus, as expected for TFs (Fig. 1B). In a final experiment, we further confirmed the interaction between CaWRKY28 

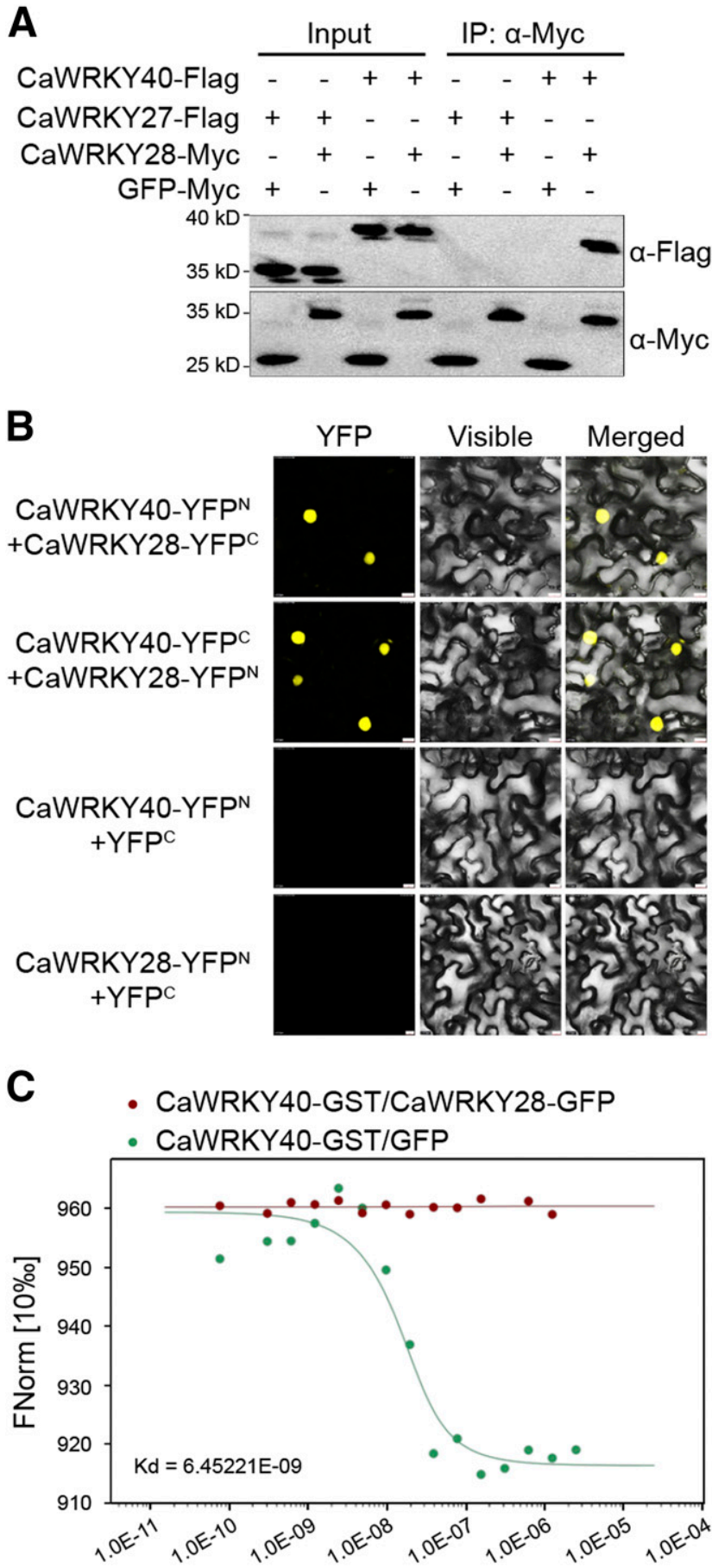

Ligand Concentration (M)

Fig. 1. CaWRKY28 interacts with CaWRKY40 in vivo and in vitro. A, Interaction between CaWRKY28 and CaWRKY40 in vivo, as determined by a coimmunoprecipitation assay. Proteins were isolated from pepper leaves transiently overexpressing CaWRKY28-Myc and CaWRKY40-FLAG, and CaWRKY40-FLAG was immunoprecipitated with anti-FLAG antibody. B, Bimolecular fluorescence complementation confirmation of the interaction between CaWRKY28 and CaWRKY40 in Nicotiana benthamiana leaves infiltrated with Agrobacterium tumefaciens strain GV3101 strain cells bearing $C a W R K Y 28-Y F P^{C}+C a W R K Y 40-Y F P^{N}$ or CaWRKY28-YFP $P^{N}+C a W R K Y 40-$ $Y F P^{C}$ constructs. YFP = yellow fluorescent protein. Yellow fluorescence, visible light, and merged images were taken by a confocal microscope at 48 $\mathrm{h}$ postinfiltration. Bars $=25 \mu \mathrm{m}$. C, Analysis of the interaction in vitro between CaWRKY28 and CaWRKY40 by microscale thermophoresis. Green fluorescent protein (GFP) or CaWRKY28-GFP:target, CaWRKY40-glutathione-S-transferase (GST):ligand. Mixtures of GFP and CaWRKY40-GST or CaWRKY28-GFP and CaWRKY40-GST were loaded onto Monolith NT.115 Capillaries and were measured using 50\% IR laser power and an LED excitation source with $\lambda=470 \mathrm{~nm}$ at ambient temperature. and CaWRKY40 by performing a microscale thermophoresis (MST) assay. We fused green fluorescent protein (GFP) to the C-terminus of CaWRKY28 to provide the fluorescent label for the method (CaWRKY28-GFP), and purified the fusion protein from protein extracts of pepper leaves transiently overexpressing CaWRKY28-GFP via Agrobacterium-mediated infiltration. In parallel, we purified CaWRKY40-GST from E. coli strain BL21 (DE3) soluble extracts. We serially diluted the purified CaWRKY40-GST fusion proteins, then mixed them with a constant amount of CaWRKY28-GFP before subjecting the mixtures to the MST assay. Based on the data from the serial dilution assay, the dissociation constant $(\mathrm{Kd})$ of the CaWRKY40GST/CaWRKY28-GFP was calculated. The CaWRKY28CaWRKY40 pair produced a clear binding curve, with a $K d$ of $6.45221 \times 10^{-09} \mathrm{~mol} /$ liter (Fig. 1C). Collectively, these results strongly argue that CaWRKY28 interacts with CaWRKY40.

Induction of CaWRKY28 expression by $R$. solanacearum infection and a subset of phytohormones in a CaWRKY40 dependent manner.

We have previously shown that CaWRKY40 is transcriptionally upregulated by $R$. solanacearum infection and by exogenous application of salicylic acid (SA), methyl jasmonate (MeJA), ethephon (ETH), or abscisic acid (ABA), and acts as positive regulator in pepper responses to $R$. solanacearum infection and to thermotolerance at high humidity (Dang et al. 2013). Because CaWRKY28 interacts with CaWRKY40, we speculated that CaWRKY28 might behave similarly to CaWRKY40 in response to the same stimuli. To test this possibility, we measured CaWRKY28 transcript levels in pepper plants challenged with $R$. solanacearum infection or subjected to an exogenous application of SA, MeJA, ETH, or ABA by real-time quantitative PCR (RT-qPCR). CaWRKY28 transcript levels rose upon $R$. solanacearum infection, as well as following SA and ETH treatment, as previously observed for CaWRKY40. However, we detected no changes after the exogenous application of MeJA or ABA, indicating that $C a W R K Y 28$ and CaWRKY4O expression patterns do not always respond to the same stimuli in the same manner (Supplementary Fig. S2). Because W-box elements are present in the promoters of both CaWRKY28 and CaWRKY40, we performed a chromatin immunoprecipitation (ChIP)-PCR assay to determine whether CaWRKY28 and CaWRKY40 might regulate the expression of their encoding genes, reciprocally or not. We used chromatin purified from pepper leaves transiently overexpressing CaWRKY28-HA or CaWRKY4O-HA with an anti-HA antibody, and discovered that CaWRKY40 directly bound to the CaWRKY28 promoter; however, the converse was not true (Supplementary Fig. S4A). We then measured CaWRKY28 and CaWRKY4O transcript levels in pepper leaves transiently overexpressing CaWRKY28 or CaWRKY40 by RT-qPCR. CaWRKY28 was upregulated by $C a W R K Y 40$ transient overexpression but CaWRKY4O was not transcriptionally regulated by CaWRKY 28 transient overexpression (Supplementary Fig. S4B), indicating that $C a W R K Y 28$ is upregulated by $R$. solanacearum in a CaWRKY40-dependent manner, because CaWRKY40 is also induced by $R$. solanacearum infection (Dang et al. 2013).

Nuclear protein CaWRKY28 acts as a positive regulator in pepper immunity against $R$. solanacearum infection.

We transiently overexpressed CaWRKY28-GFP in N. benthamiana leaves by Agrobacterium-mediated infiltration. We visualized GFP fluorescence at $48 \mathrm{hpi}$ using a confocal microscope (TCS SP8; Leica Microsystems, Germany). We observed free GFP, derived from 35S:GFP, in the cytoplasm and nuclei of infiltrated epidermal cells, whereas GFP fluorescence for CaWRKY28-GFP was restricted to the nucleus (Supplementary Fig. S3), indicating that CaWRKY28 is a nuclear protein. 
Because CaWRKY28 is regulated by $R$. solanacearum infection, we reasoned that it might be involved in pepper immunity against the bacterium. To test this hypothesis, we studied the effect of virus-induced CaWRKY28 silencing on pepper immunity against RSI. We constructed a vector with a specific fragment in the $3^{\prime}$ untranslated region (UTR) of CaWRKY28 (approximately $200 \mathrm{bp}$ in length), whose specificity was confirmed by blasting against the genome of pepper. With this vector, we successfully and specifically silenced CaWRKY28 in pepper plants, achieving an over $90 \%$ reduction in CaWRKY 28 transcript levels, while maintaining normal transcript levels of CaWRKY71, which exhibits the highest sequence similarity to CaWRKY28 among all of the pepper WRKY genes (Fig. 2A). Upon challenge by $R$. solanacearum infection, CaWRKY28silenced pepper plants exhibited an increased susceptibility to the $R$. solanacearum, as indicated by the more severe bacterial wilt symptoms and greater $R$. solanacearum population growth (in CFU) in these silenced plants as compared with the control plants. Consistent with these results, genes encoding pathogenesis-related (PR) proteins such as CaPRl (Pan et al. 2010; Zhang et al. 2012), NONEXPRESSER OF PR GENES 1 (CANPRI) (Chen et al. 2009; Zhang et al. 2012), DEFENSIN 1 (CaDEF1) (Choi et al. 2008, 2015; Kim et al. 2015; Zhang et al. 2019), and ABSCISIC ACID-RESPONSIVE 1 ( $C a A B R l)$ (Choi and Hwang 2011) showed much lower expression levels in CaWRKY28-silenced pepper plants relative to mock-treated control plants (Fig. 2). The SRDX domain was used to transform CaWRKY28 into dominant-negative repressor versions and has been widely used to assess the roles of TFs (Grunewald et al. 2013; Takada 2013). In comparison, we characterized the effects associated with the transient overexpression of 35S:CaWRKY28$H A$ or its chimeric repressor version 35S:CaWRKY28-SRDX-HA on hypersensitive reaction (HR)-like cell death and the transcript levels of immunity-associated genes. We infiltrated A. tumefaciens strain GV3101 cells bearing the 35S:CaWRKY28-HA or 35S:CaWRKY28-SRDX-HA construct (35S:HA acting as negative control) into the leaves of pepper plants. We first confirmed that the constructs, indeed, led to the accumulation of the tagged CaWRKY28 by performing RT-qPCR (for the transcripts) and immunoblot analysis with protein extracts from pepper leaves transiently overexpressing CaWRKY28-HA or CaWRKY28$S R D X-H A$ using an anti-HA antibody (for the proteins). Both CaWRKY28-HA and CaWRKY28-SRDX-HA appeared to successfully accumulate in pepper leaves (Supplementary Fig. S5A). Overexpression of CaWRKY28-HA caused extensive HR-like cell death, accompanied by increased leaf ion leakage, as illustrated by higher conductivity. By contrast, neither CaWRKY28-SRDX$H A$ nor the control triggered cell death (Supplementary Fig. S5B to D). In agreement, the transient overexpression of CaWRKY28$H A$ significantly raised the transcript levels of CaPRl, CaNPR1, $C a D E F 1$, and $C a A B R 1$, whereas the transient overexpression of CaWRKY28-SRDX-HA had the opposite effect, when compared
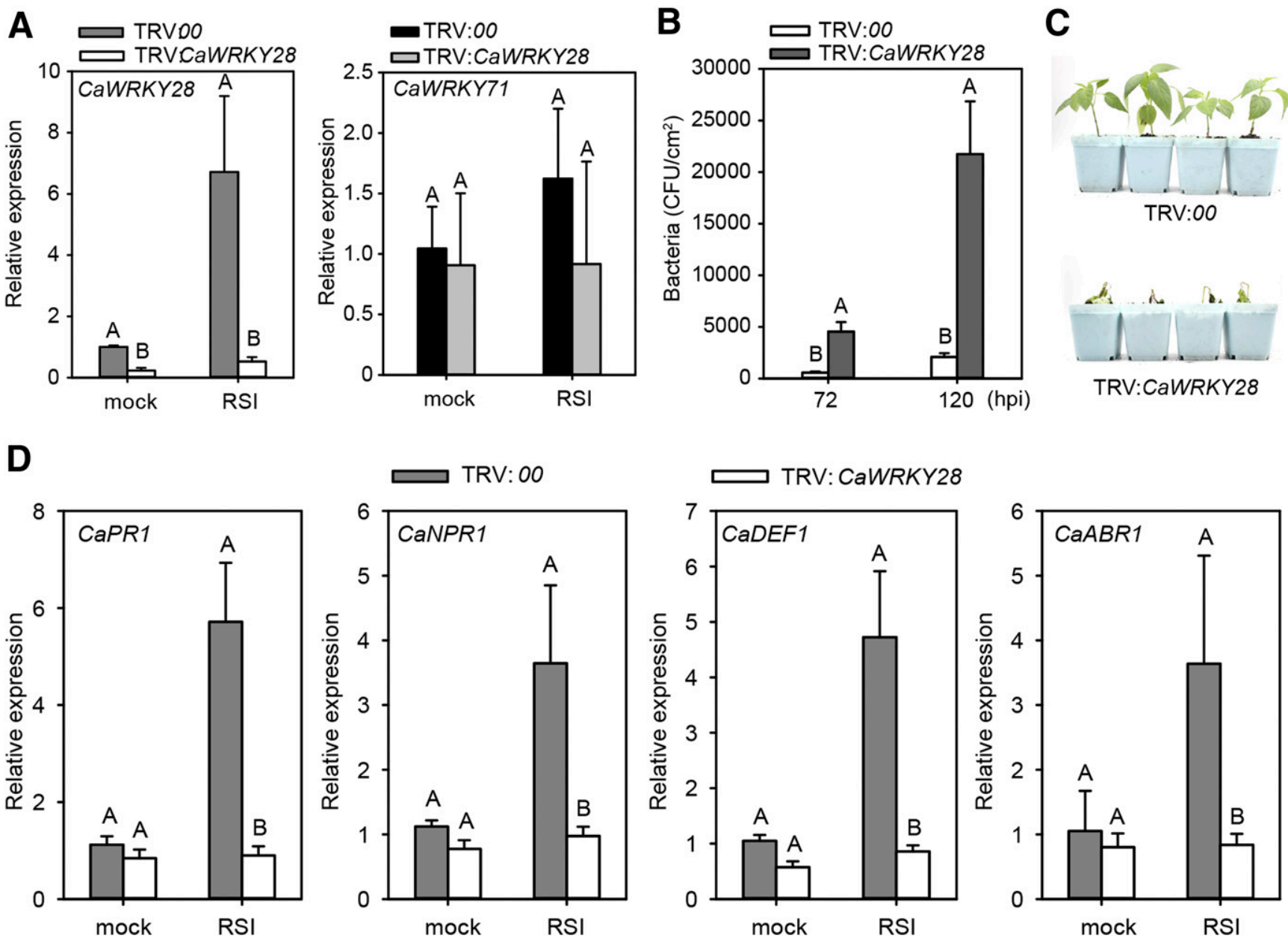

Fig. 2. Effect of CaWRKY28 silencing on the response of pepper plants to Ralstonia solanacearum infection. A, Relative transcript levels of CaWRKY28 and CaWRKY71 in TRV:CaWRKY28 pepper plants challenged with $R$. solanacearum infection (RSI). B, Effect of CaWRKY28 silencing on R. solanacearum growth, shown as CFU; hpi $=$ hours postinfiltration. C, Bacterial wilt symptoms of pepper plants (CaWRKY28-silenced and control) challenged by RSI at 7 days postinoculation (dpi). D, Relative transcript levels of the immunity-associated genes CaPR1, CaNPR1, CaDEF1, and CaABR1 in pepper plants (CaWRKY28-silenced and control) challenged by RSI. Data are shown as means \pm standard error of four replicates (A, B, and D). Different uppercase letters above the bars indicate significant differences between means $(P<0.01)$ by Fisher's protected least significant difference test. 
with the control plants (Supplementary Fig. S5E). All of these results indicate that CaWRKY28 acts positively in pepper responses to $R$. solanacearum infection.

\section{Dual transient overexpression of CaWRKY28 and CaWRKY40 additively triggers defense response in pepper leaves.}

To determine the effect of the interaction between CaWRKY28 and CaWRKY40 on pepper immunity, we transiently overexpressed both CaWRKY28-HA and CaWRKY40-HA in pepper plant leaves, and recorded the consequences of their dual transient overexpression on pepper defense response. We first confirmed that the two proteins successfully accumulated by immunoblot analysis with an anti-HA antibody (Supplementary Fig. S6A), and that the cotransient accumulation of CaWRKY28 and $C a W R K Y 40$ induced a stronger HR-like cell death response (Supplementary Fig. S6B), more pronounced ion leakage as measured by conductivity (Supplementary Fig. S6C), as well as higher transcript levels of CaPR1, CaNPR1, CaDEF1, and $C a A B R 1$, when compared with the transient accumulation of CaWRKY28 or CaWRKY40 alone (Supplementary Fig. S6D). Therefore, these results suggest that CaWRKY28 and CaWRKY40 synergistically act in pepper immunity.

\section{CaWRKY40 silencing blocks CaWRKY28-mediated} immunity but the converse is not true.

To further catalog the effects linked to the functional association of CaWRKY28 and CaWRKY40 during pepper immunity, we measured the effects of silencing CaWRKY4O on the HR-like cell death triggered by transient overexpression of CaWRKY28$H A$. We first determined that CaWRKY28 successfully accumulated during CaWRKY28-HA transient overexpression in pepper leaves and that CaWRKY4O was efficiently silenced by virusinduced gene silencing (VIGS), by carrying out an immunoblot analysis with an anti-HA antibody (for the detection of CaWRKY28 protein) and by RT-qPCR (for CaWRKY28 transcripts) (Fig. 3A and C). The transient overexpression of CaWRKY28-HA in leaves triggered intensive HR-like cell death in the control plants (with normal CaWRKY4O levels) but not in the CaWRKY40-silenced plants (Fig. 3B). Consistent with these observations, transient overexpression of CaWRKY28-HA in the leaves of control plants led to upregulations of the immunityrelated genes CaPR1, CaNPR1, CaDEF1, and CaABR1; however, these upregulations were almost completely abolished upon CaWRKY4O silencing (Fig. 3C). Taking a reciprocal approach, silencing CaWRKY28 significantly attenuated but did not completely block CaWRKY40 function in pepper immunity (Fig. 3B and C). These results indicate that CaWRKY28 functions in pepper immunity in a CaWRKY40-dependent manner, while the function of CaWRKY40 is potentiated by CaWRKY28.

\section{CaWRKY28 targets and regulates CaPR1, CaNPR1, $C a D E F 1$, and $C a A B R 1$ in planta in a CaWRKY40-dependent manner.}

Because CaWRKY28 acts as a regulator of the expression of CaPR1, CaNPR1, CaDEF1, and CaABR1 (Supplementary Figs. S5E and S6D), whose promoters all contain more than one W-box, it stands to reason that CaWRKY28 might regulate these genes directly. To test this possibility, we performed a ChIP-PCR assay using chromatin isolated from pepper leaves infiltrated with $A$. tumefaciens strain GV3101 containing 35S:CaWRKY28-HA at 48 hpi and appropriate primers of $\mathrm{W}$-box containing promoter fragments. All primer pairs produced clear PCR products, while primer pairs designed against another promoter fragment lacking the W-box did not result in a product (Fig. 3D), indicating that CaWRKY28 likely binds to the promoters of the four immunityrelated genes tested here in a W-box-dependent manner. To determine whether CaWRKY40 might contribute to the targeting of these target genes by CaWRKY28, we isolated chromatin from CaWRKY40-silenced pepper leaves transiently overexpressing CaWRKY28-HA for use as template for ChIP-PCR. Satisfyingly, CaWRKY4O silencing almost completely blocked the enrichment of CaWRKY28 at the tested promoters. Using the reciprocal approach, we discovered that silencing CaWRKY 28 significantly decreased but did not block the enrichment of CaWRKY40 at the promoters of the tested $P R$ genes, whether or not pepper plants were challenged by $R$. solanacearum infection (Fig. 3E and F). Based on these results, we hypothesize that CaWRKY28 may fulfill its function in pepper immunity by potentiating CaWRKY40 to directly target and regulate immunity-related genes such as CaPR1, CaNPR1, CaDEF1, and CaABR1.

\section{Cys249 is required for the function of CaWRKY28 in HR-like cell death induction.}

Although it belongs to the group II WRKY proteins, CaWRKY28 also shares characteristics of group III WRKYs due to the presence of the Cys249 residue, which is found only in CaWRKY28 orthologs from the Solanaceae family, and is replaced by isoleucine (Ile) or threonine (Thr) in its Arabidopsis and rice orthologs, respectively. This conserved residue piqued our interest. To test whether this residue played a role in CaWRKY28 function during the regulation of pepper immunity, we replaced Cys249 (UGC) with Thr, as in Arabidopsis, generating CaWRKY $28^{\mathrm{C} 249 \mathrm{~W}}$ (Fig. 4A). We infiltrated pepper leaves with $A$. tumefaciens strain GV3101 cells carrying 35S:CaWRKY28-HA, 35S:CaWRKY $28^{C 249 W}-H A$, or 35S:HA (as control) constructs. The transient overexpression of CaWRKY28-HA triggered intensive HR-like cell death, as expected, whereas neither CaWR$K Y 28^{C 249 W}-H A$ transient overexpression nor the control resulted in cell death (Fig. 4B). Just like CaWRKY28, the mutated version CaWRKY28C249W accumulated in the nucleus, indicating that Cys249 does not contribute to the subcellular localization of CaWRKY28 (Fig. 4C). In addition, we tested the binding of CaWRKY $28^{\mathrm{C} 249 \mathrm{~W}}$ to the W-box in an electrophoretic mobility shift assay (EMSA) by using a CaWRKY28 ${ }^{\mathrm{C} 249 \mathrm{~W}}$-GST (Fig. 4D), wild-type probe, mutated probe, and unlabeled probe (Fig. 4E). We observed a clear interaction (as indicated by the mobility shift) between CaWRKY28-GST or CaWRKY28 ${ }^{\mathrm{C} 249 \mathrm{~W}}$-GST and the W-box-containing probe but not to the mutated probe (Fig. 4F), indicating that CaWRKY $28^{\mathrm{C} 249 \mathrm{~W}}$ retains the ability to bind the Wbox in vitro. To further characterize the role of the Cys249 residue in the binding of CaWRKY28 to the W-box in planta, we performed a ChIP-PCR assay to compare the binding affinity of CaWRKY28 and CaWRKY28 $249 \mathrm{~W}$ to the CaPRl, CaNPRl, $C a D E F 1$, and $C a A B R 1$ promoters, using chromatin isolated from pepper leaves transiently overexpressing CaWRKY28 or CaWR$K Y 28^{C 249 W}$. Primer pairs for all four tested genes produced clear PCR products when using chromatin isolated from pepper leaves by transient overexpression of CaWRKY28 but not CaWR$K Y 28^{C 249 W}$, indicating that CaWRKY $28^{\mathrm{C} 249 \mathrm{~W}}$ cannot bind to the $\mathrm{W}$-box in vivo (Supplementary Fig. S7A). In agreement with this observation, transient overexpression of CaWRKY28 increased the transcript levels of the four immunity-related genes but transient overexpression of CaWRKY28 $8^{C 249 W}$ did not (Supplementary Fig. S7B). Together, these data indicate that Cys249 plays a role in the function of CaWRKY28 during pepper immunity.

\section{CaWRKY28-CaWRKY40 interaction, mediated} by Cys249, prevents CaWRKY28

from binding the $\mathrm{W}$-box in planta.

To further clarify the possible role of Cys249 in CaWRKY28 function in the context of pepper immunity, we assayed the 
A

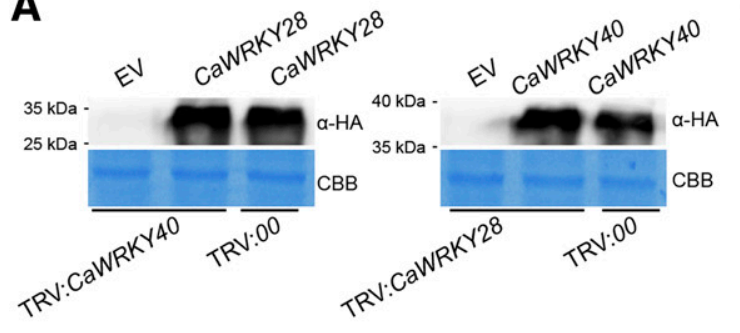

B

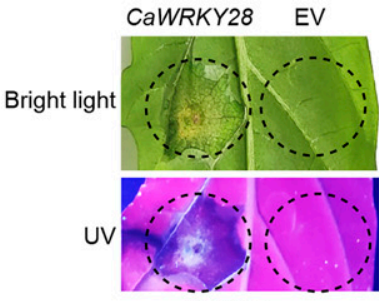

TRV:00

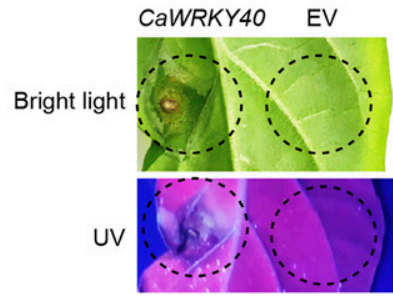

TRV:00
CaWRKY28 EV

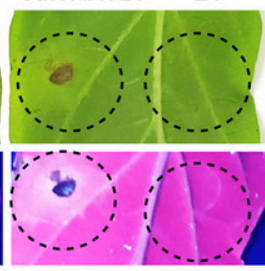

TRV:CAWRKY40

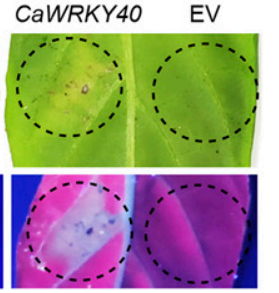

TRV:CaWRKY28

C
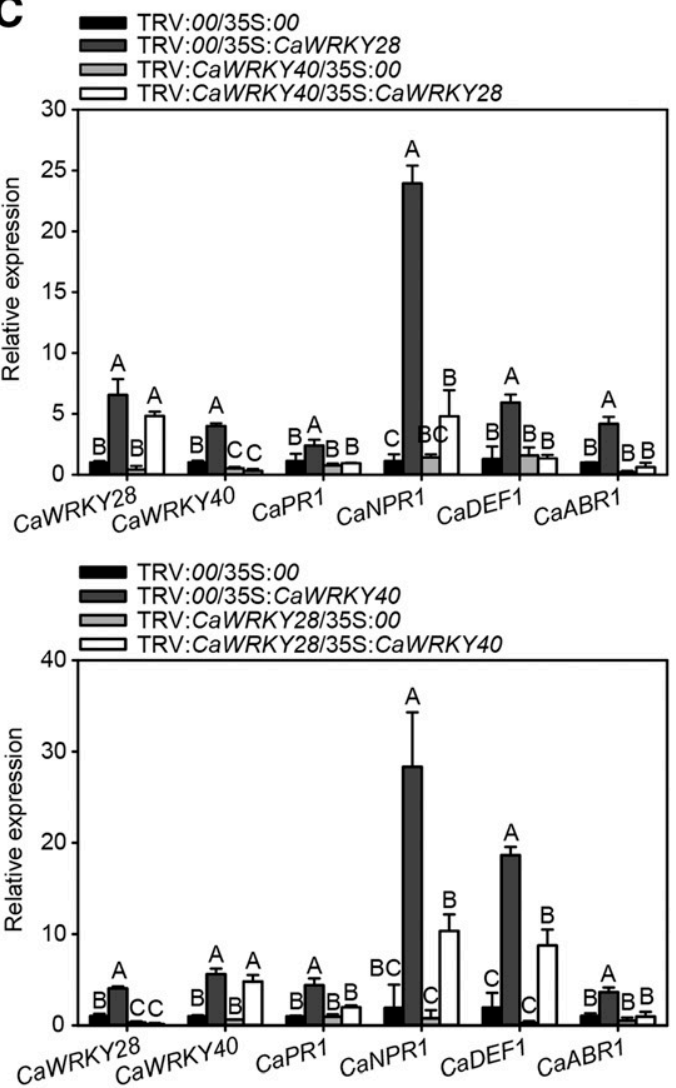

D

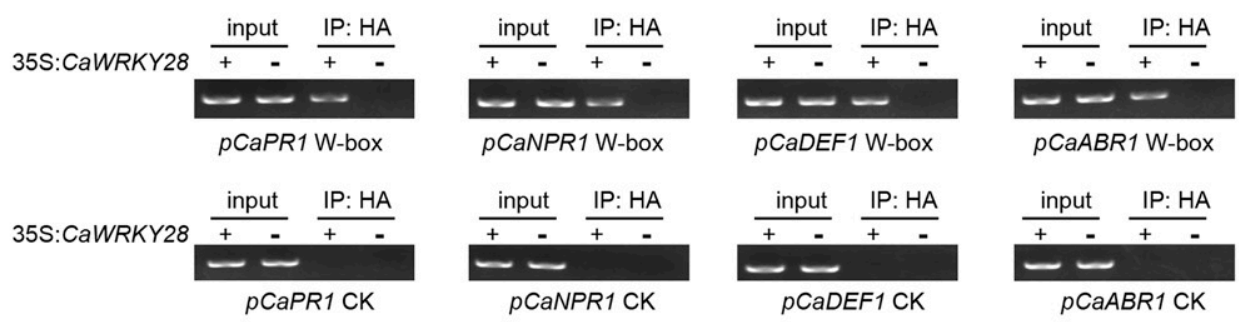

E

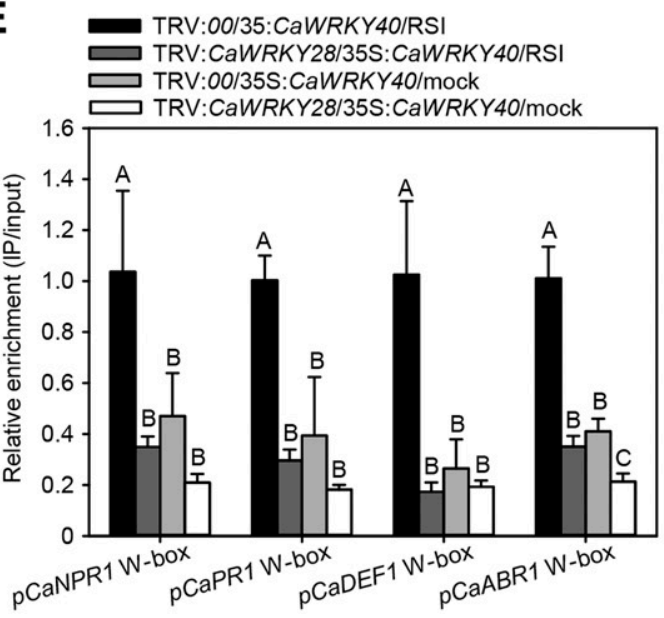

F
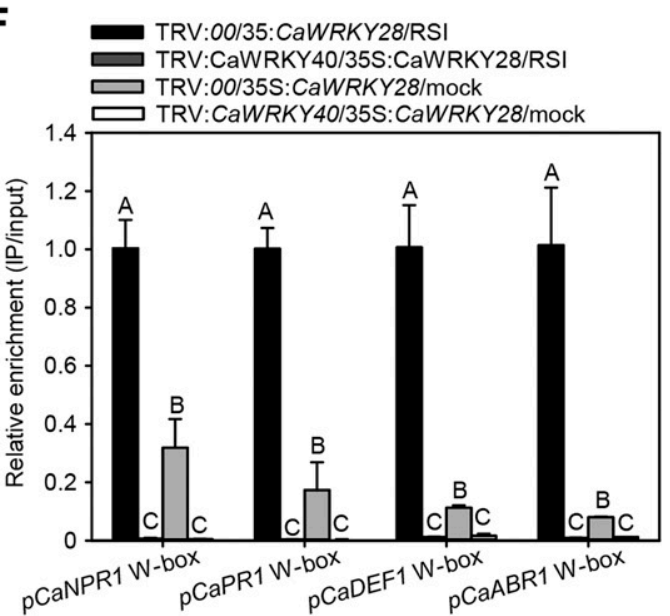

Fig. 3. CaWRKY28 and CaWRKY40 both contribute to regulating pepper immunity. A, Accumulation of CaWRKY28-HA and CaWRKY40-HA in pepper leaves transiently overexpressing CaWRKY28-HA or CaWRKY40-HA, in the context of wild-type plants (pTRV:00) or plants silenced for CaWRKY28 (pTRV:CaWRKY28) or CaWRKY40 (pTRV:CaWRKY40), by immunoblot analysis with anti-HA antibody. EV = empty vector. B, Effect of CaWRKY28 or CaWRKY4O silencing on hypersensitive reaction-like cell death phenotypes induced by transient overexpression of CaWRKY28 or CaWRKY4O. C, Effect of CaWRKY28 or CaWRKY4O silencing in pepper plants transiently overexpressing CaWRKY28 or CaWRKY4O on the relative transcript levels of immunity-related genes. D, Enrichment of CaWRKY28 at the promoters of immunity-related marker genes, as determined by chromatin immunoprecipitation PCR. E, Effect of CaWRKY28 silencing on the enrichment of CaWRKY40 at the promoters of immunity-related marker genes. F, Effect of CaWRKY40 silencing on the enrichment of CaWRKY28 at the promoters of immunity-related marker genes. Enrichment of the control was set to 1 after normalization by input (E and F). Data are shown as means \pm standard error of four replicates $(\mathrm{C}, \mathrm{E}$, and $\mathrm{F})$. Different uppercase letters above the bars indicate significant differences between means $(P<0.01)$ by Fisher's protected least significant difference test. 
interaction between CaWRKY28 $249 \mathrm{~W}$ or CaWRKY28 and CaWRKY40 by BiFC assay. CaWRKY28 interacted with CaWRKY40, while CaWRKY28 ${ }^{\mathrm{C} 249 \mathrm{~W}}$ did not (Fig. 5A). To further confirm this result, we performed an MST assay with CaWRKY40-GST purified from E. coli strain BL21 (DE3) and CaWRKY28-GFP or CaWRKY $28^{\mathrm{C} 249 \mathrm{~W}}$-GFP (isolated from the leaves of pepper plants transiently overexpressing CaWRKY28$G F P$ or $C a W R K Y 28^{C 249 W}$-GFP, respectively, by immunoprecipitation with an anti-GFP antibody). Our results showed that CaWRKY28-GFP and CaWRKY40-GST produced a clear binding curve, consistent with physical interaction of the two proteins, but CaWRKY28 $8^{\mathrm{C} 249 \mathrm{~W}}$-GFP and CaWRKY40-GST did not (Fig. 5B). These data indicate that Cys249 is crucial for the interaction of CaWRKY28 and CaWRKY40.

To determine whether CaWRKY40 might influence the DNA binding activity of CaWRKY28 by the formation of a CaWRKY28-CaWRKY40 complex, we turned to EMSA with CaWRKY40m, a mutant version of CaWRKY40 that cannot bind to the W-box (Shen et al. 2020). Using CaWRKY28 ${ }^{\mathrm{C} 249 \mathrm{~W}}$-GST purified from $E$. coli strain BL21 (DE3) and the W-box-containing wildtype probe, we detected a mobility shift when CaWRKY28 ${ }^{\mathrm{C} 249 \mathrm{~W}}$. GST was incubated with excess CaWRKY40, indicating that CaWRKY28 ${ }^{\mathrm{C} 249 \mathrm{~W}}$ can bind to the W-box (Fig. 5C), although it does not interact with CaWRKY40 (Fig. 5B). By contrast, we failed to detect a mobility shift when CaWRKY28 was presented with the W-box-containing wild-type probe and incubated with excess CaWRKY40m, although both recombinant proteins do interact (Fig. 5C; Supplementary Fig. S8). These results indicate that the interaction between CaWRKY28 and CaWRKY40, which is determined by Cys249 in CaWRKY28, prevents CaWRKY28 from binding to the W-box containing DNA sequences. To test the effect of the CaWRKY28-CaWRKY40 interaction on the DNA binding potential of CaWRKY28, we next compared the effect of $C a W R K Y 28$ (or $C a W R K Y 28^{C 249 W}$ ) transient overexpression with or without CaWRKY4O transient overexpression in CaWRKY40silenced pepper leaves on the enrichment of CaWRKY40 at the CaPRl, CaNPRl, CaDEF1, and CaABRl promoters by ChIPqPCR. Note that the VIGS construct targets 3'UTR of CaWRKY40; therefore, it is possible to overexpress CaWRKY4O from a heterologous construct bearing a foreign transcription terminator. The enrichment of CaWRKY28 at the promoters of the four tested genes was increased by cotransient overexpression of CaWRKY4O and CaWRKY 28 but not by cotransient overexpression of CaWRKY4O and CaWRKY $28^{C 249 W}$ (Fig. 5D), indicating that the targeting of CaWRKY28 to the four tested immunity-related genes is potentiated by CaWRKY40 when the two proteins interact.

\section{Modulation of thermotolerance-related $\mathrm{CaHSP24}$} by CaWRKY40 is not regulated by CaWRKY28.

Our results thus far show that CaWRKY40 acts as a positive regulator of pepper immunity and thermotolerance (Dang et al. 2013); CaWRKY40 function during infection by $R$. solanacearum is positively modulated by CaWRKY28, inviting the following question: does CaWRKY28 also modulate the function of CaWRKY40 in the regulation of thermotolerance? Our results showed that transient overexpression of CaWRKY28-HA did not alter the expression of the small heat shock protein gene CaHSP24 (Supplementary Fig. S9A). Conversely, the silencing
A

CaWRKY28 241 ---YEGQHNHHCPAALRGN--- 256

CaWRKY28 ${ }^{\mathrm{C} 249 \mathrm{~W}} 241$---YEGQHNHHWPAALRGN--- 256

B

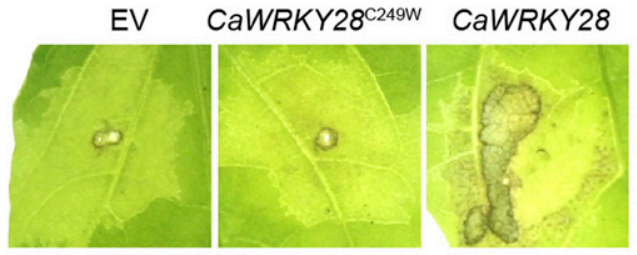

C

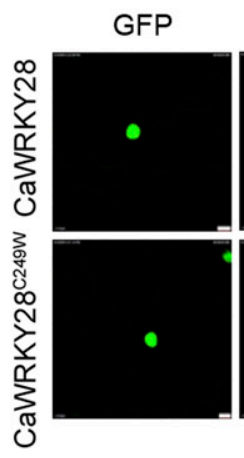

DAPI
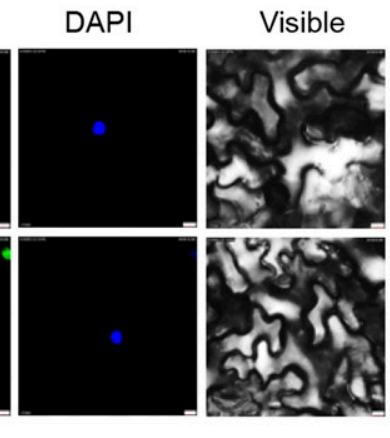

Merged

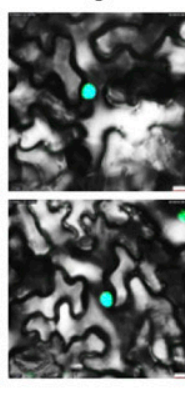

D

Wild-type probe GCTTTGATACCAAGTTTTTCTTGACCCAAA

Mutated probe GCTTTGATACCAAGTTTTTCTGGGGCCAAA
$\mathbf{E}$

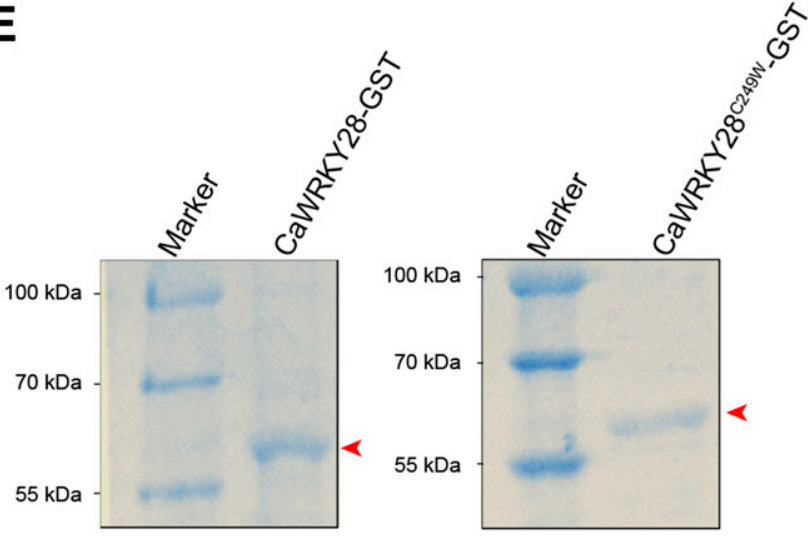

$\mathbf{F}$

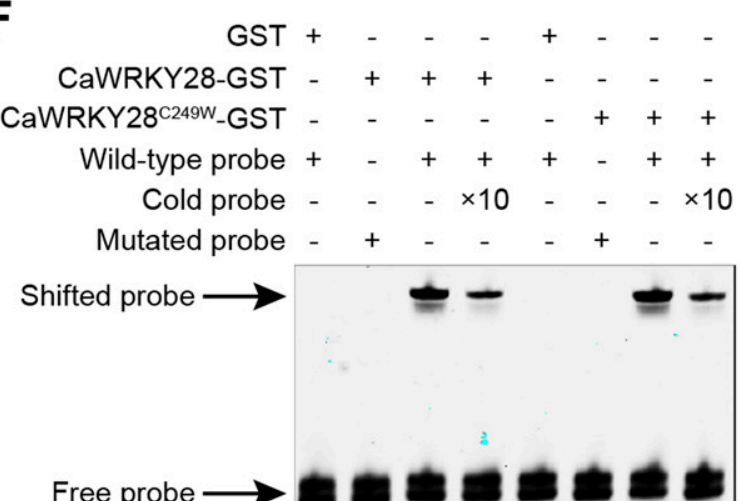

Fig. 4. Cys249 in CaWRKY28 is necessary for the regulation of pepper immunity. A, Cys249 (codon UGC) in CaWRKY28 was mutated to Trp249 (codon UGG) (CaWRKY28 $249 \mathrm{~W}$ ). B, Hypersensitive reaction-like cell death phenotype triggered by transient overexpression of CaWRKY28 and CaWR$K Y 28^{C 249 W}$ at $48 \mathrm{~h}$ postinfiltration. EV = empty vector. C, Nuclear localization of the CaWRKY28 $249 \mathrm{~W}$ in Nicotiana benthamiana epidermal cells. GFP = green fluorescent protein and DAPI = 4',6-diamidino-2-phenylindole. D, Wild-type probe and its mutant version for electrophoretic mobility shift assay (EMSA). E, Purification of CaWRKY28-glutathione-S-transferase (GST) and CaWRKY28 249 -GST protein. F, Binding of CaWRKY28 and CaWRKY $28^{\mathrm{C} 249 \mathrm{~W}}$ to W-box-containing probe by EMSA. 
of CaWRKY28 did not affect the upregulation of CaHSP24 by transient overexpression of CaWRKY40-HA (Supplementary Fig. S9B). We also did not observe any synergistic effect on CaHSP24 transcript levels when both CaWRKY28-HA and CaWRKY40-HA were transiently overexpressed, relative to CaWRKY40-HA transient overexpression alone (Supplementary Fig. S9C). These data indicate that CaWRKY28 does not potentiate CaWRKY40 in its modulation of CaHSP24 expression.

\section{DISCUSSION}

The majority of WRKY family members have been implicated in immune responses against attacks from various pathogens in different plant species (Dong et al. 2003; Pandey and Somssich 2009; Segonzac et al. 2017). WRKYs have been suggested to organize into interconnected WRKY networks (Eulgem and Somssich 2007). However, how these WRKY TFs build such networks and how these networks operate to produce appropriate transcriptional outputs remain poorly understood. In the present study, we provide evidence that, by interacting with CaWRKY40, CaWRKY28 acts as a positive regulator in pepper response to $R$. solanacearum infection by potentiating the targeting and regulation of immunity-related genes by CaWRKY40, rather than by directly targeting immunity genes on its own. Moreover, Cys249 in CaWRKY28 is critical for its interaction with CaWRKY40.

\section{CaWRKY28 acts as positive regulator}

in pepper immunity during $R$. solanacearum infection.

The evidence that CaWRKY28 acts as a positive regulator in pepper immunity in response to $R$. solanacearum infection

A

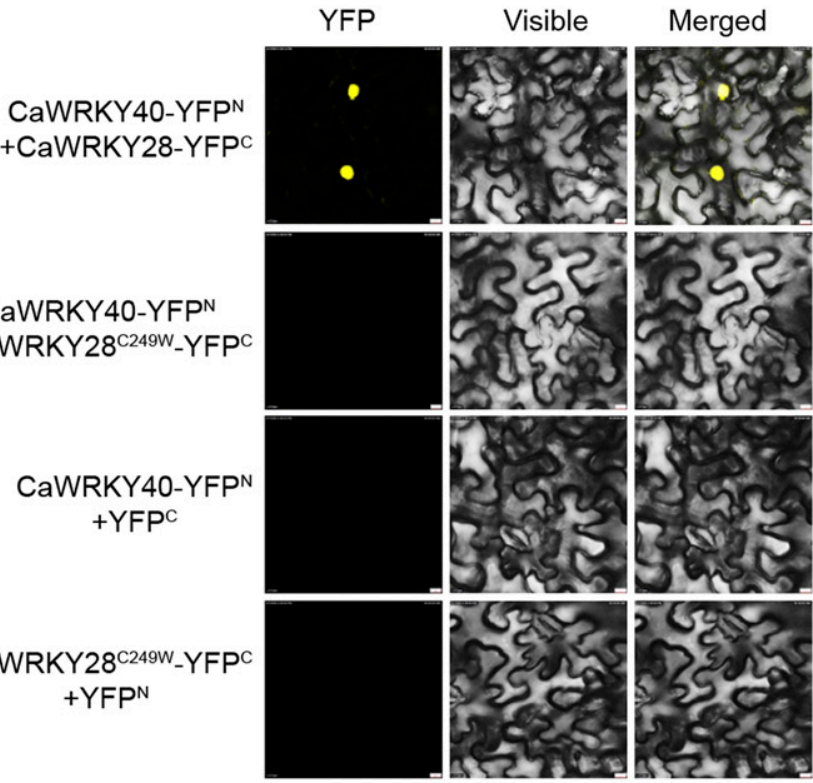

B

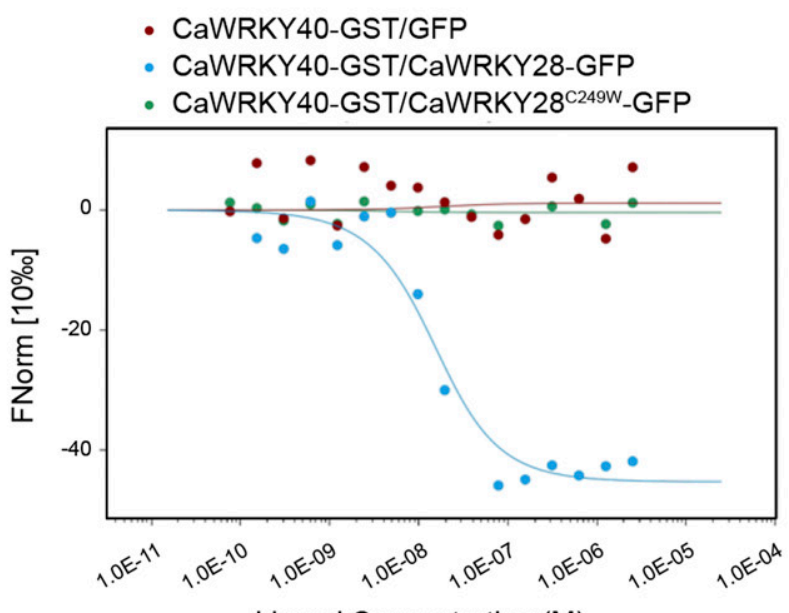

C

CaWRKY40 175 --GYQWRKYGQKVTRD-- 189

CaWRKY40-m 175 --GYQWRKYGMKVTRD-- 189
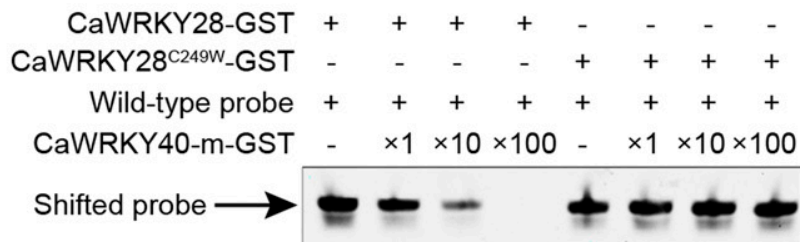

Free probe $\rightarrow 00000000$

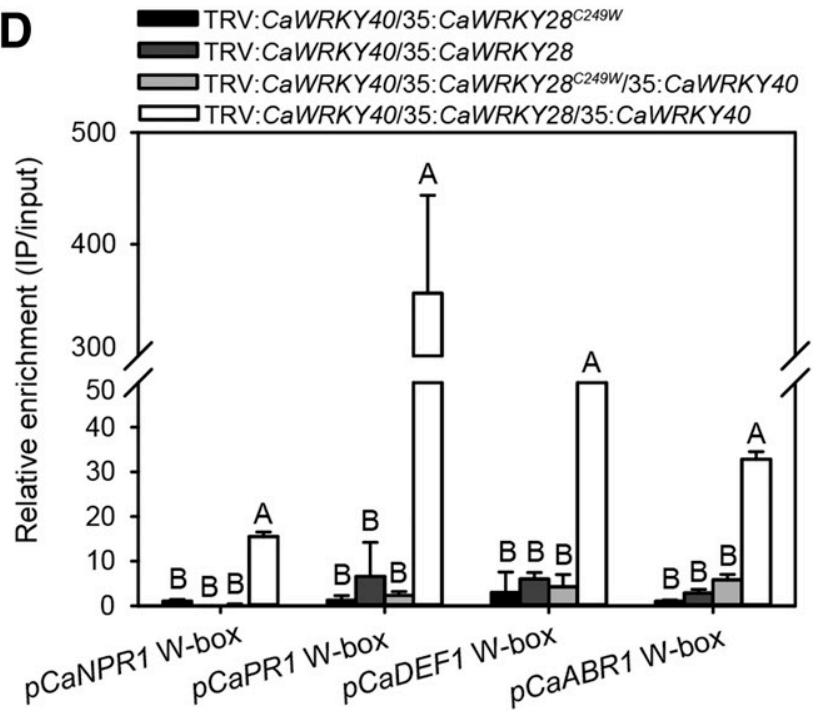

Fig. 5. CaWRKY28 Cys249 is critical for the protein's interaction with CaWRKY40, DNA binding, and transcriptional activation of target genes. A, Bimolecular fluorescence complementation assay of the interaction between CaWRKY28 or CaWRKY28 ${ }^{\mathrm{C} 249 \mathrm{~W}}$ and CaWRKY40 in Nicotiana benthamiana leaves. YFP $=$ yellow fluorescent protein. B, Microscale thermophoresis assay of the interaction between CaWRKY28 or CaWRKY28 ${ }^{\mathrm{C} 249 \mathrm{~W}}$ and CaWRKY40 in solution. Green fluorescent protein (GFP), CaWRKY28-GFP, CaWRKY28 ${ }^{\mathrm{C} 249 \mathrm{~W}}$-GFP:targets; CaWRKY40-glutathione- $S$-transferase (GST):ligand. C, Electrophoretic mobility shift assay of the binding of CaWRKY28-GST and CaWRKY28 $249 \mathrm{~W}$-GST to the W-box-containing probe, with or without CaWRKY40-m (Shen et al. 2020). D, Enrichment of CaWRKY28-GFP or CaWRKY28 ${ }^{\mathrm{C} 249 \mathrm{~W}-\mathrm{GFP}}$ at the promoters of immunity-related marker genes by chromatin immunoprecipitation quantitative PCR (qPCR) assay in pepper plants silenced for CaWRKY4O and transiently overexpressing CaWRKY28-GFP or CaWRKY28C249W- GFP, with or without cotransient overexpression of CaWRKY40-GFP. For qPCR assays, CaWRKY28-GFP (or CaWRKY $28^{C 249 W-G F P)}$ was cotransiently overexpressed with CaWRKY40-GFP (or empty vector as control) in the leaves of CaWRKY40-silenced pepper plants. The enrichment of the control was set to 1 after normalization by input. Data are shown as means \pm standard error of four replicates. Different uppercase letters above the bars indicate significant differences between means $(P<0.01)$ by Fisher's protected least significant difference test. 
comes from the following observations. First, CaWRKY28 is upregulated by $R$. solanacearum infection and by exogenous applications of SA and ETH. The signaling role mediated by SA or ET during plant immunity has been well established (Boller and Felix 2009; Cui et al. 2015; Tsuda and Katagiri 2010). Second, the silencing of CaWRKY28 significantly raised the susceptibility of pepper plants to $R$. solanacearum infection. By contrast, transient overexpression of CaWRKY28 triggered HRlike cell death, consistent with an increased resistance to pathogen attack. Third, transcript levels of the immunity-associated genes CaPRl (Pan et al. 2010; Zhang et al. 2012), CaNPRl (Chen et al. 2009; Zhang et al. 2012), CaDEF1(Choi et al. 2008, 2015; Kim et al. 2015; Zhang et al. 2019), and CaABRI (Choi and Hwang 2011) were significantly downregulated by CaWRKY28 silencing, while upregulated by CaWRKY28 transient overexpression in pepper plants. We may speculate that, upon $R$. solanacearum infection, CaWRKY 28 expression is upregulated, thereby activating immunity-associated genes leading to enhanced resistance to $R$. solanacearum infection. Thus far, a subset of WRKY TFs such as CaWRKY6 (Cai et al. 2015), CaWRKY22 (Hussain et al. 2018), CaWRKY27 (Dang et al. 2014), CaWRKY40 (Dang et al. 2013), CaWRKY40b (Ifnan Khan et al. 2018), and CaWRKY58 (Wang et al. 2013) have been implicated in pepper immune responses to $R$. solanacearum infection, acting as positive or negative regulators. Their individual silencing produced distinctive phenotypes related to immune responses to $R$. solanacearum infection that are reminiscent of pathogen-associated molecular pattern-triggered immunity, with synergistic relationships between the signaling components (Tsuda et al. 2009).

\section{CaWRKY28 interacts with and potentiates CaWRKY40} in regulating immunity against $R$. solanacearum infection but not thermotolerance.

Previous work has shown that the functions of WRKY TFs can be modulated by other proteins, including WRKYs, by direct protein-protein interaction (Chi et al. 2013), defining WRKY networks in plant defense signaling (Eulgem and Somssich 2007). An interaction between WRKY TFs might be necessary for their cooperative or antagonistic transcriptional regulation, by binding to closely spaced or distantly separated W-boxes in the promoters of their target genes, which is consistent with the observation that, thus far, only group II and group III WRKY proteins (with a single WRKY domain) have been shown to interact with other WRKYs (Chi et al. 2013). Herein, we described a novel type of WRKY interaction between CaWRKY28 and CaWRKY40, which was originally discovered by pull-down followed by LC-MS/MS and further confirmed by Co-IP, BiFC, and MST assays. Although CaWRKY28 can bind to the W-box in vitro, as indicated by results from EMSA (Fig. $5 \mathrm{C}$ ), this binding might be prevented by CaWRKY40 via interaction with CaWRKY28 in vivo (Fig. 5C). We also revealed that the targeting and the regulation of immunity-related genes (CaPRl, CaNPRl, CaDEF1, and CaABRl) by CaWRKY40 was enhanced by interaction with $C a W R K Y 28$, whereas the regulation of the thermotolerance-related gene CaHSP24 was not affected by CaWRKY28 (Dang et al. 2013). Because WRKYs such as CaWRKY40 have been frequently found to be involved in regulating several seemingly disparate processes (Nuruzzaman et al. 2013; Rushton et al. 2010), we speculate that these TFs might achieve their specificity of function via a similar mechanism, through an interaction with appropriate transcriptional regulators such as CaWRKY28. Noticeably, although CaWRKY28 and CaWRKY40 transcript levels responded similarly to exogenous application of SA and ETH (Dang et al. 2013), they differed in their response to exogenously applied ABA and MeJA. This difference might provide the CaWRKY28-CaWRKY40 module with great regulatory adaptability against different internal and external stimuli.

\section{Cys249 in CaWRKY28 is critical for the interaction of CaWRKY28 with CaWRKY40.}

In previous studies, protein-protein interactions between WRKY proteins were thought to be mediated by their leucine zipper domain (Chi et al. 2013). However, our results indicate that the Cys249 residue within CaWRKY28 is critical for its interaction with CaWRKY40, because CaWRKY28 ${ }^{\mathrm{C} 249 \mathrm{~W}}$ lost its ability to interact with CaWRKY40, thereby losing its W-box binding in vivo as well as its ability to induce the immune response. Interestingly, we determined that CaWRKY28 $8^{\mathrm{C} 249 \mathrm{~W}}$ was able to bind W-box-containing promoters from immunity-related genes in vitro but not in vivo. Because CaWRKY28 ${ }^{\mathrm{C} 249 \mathrm{~W}}$ cannot interact with CaWRKY40, we can exclude any blocking effect of CaWRKY40 on the binding of CaWR$\mathrm{KY} 28^{\mathrm{C} 249 \mathrm{~W}}$ to W-box containing promoters in vivo. Therefore, we speculate that interacting proteins other than CaWRKY40 might prevent CaWRKY $28^{\mathrm{C} 249 \mathrm{~W}}$ from binding to W-box containing promoters. In addition, it appears that the interaction between WRKY28 and WRKY40 family members is unique to the Solanaceae family, because the Cys249 residue is shared only across CaWRKY28 orthologs from the Solanaceae species tobacco, tomato, and potato but not from other plant species such as rice or Arabidopsis. It is generally accepted that a given gene and its orthologs, as well as the underlying protein-protein interactions between WRKY TFs, can vary across plant species (Abeysinghe et al. 2019; Chakraborty et al. 2018; Dang et al. 2013). For example, whereas WRKY40 facilitates powdery mildew infection in Arabidopsis (Pandey et al. 2010) and acts as a negative regulator to prevent exaggerated defense responses (Birkenbihl et al. 2017a), its orthologs in pepper and in chickpea (Cicer arietinum) act as positive regulators of the immune response to different pathogens (Chakraborty et al. 2018; Dang et al. 2013). We speculate that the specific presence of Cys249 in CaWRKY28, and the specific interaction between WRKY28 and WRKY40, might contribute to the different roles of WRKY40 TFs in different plant species.

Collectively, our data indicate that, by interacting with CaWRKY40, CaWRKY28 acts as a positive regulator in pepper response to $R$. solanacearum infection. CaWRKY28 does not directly target promoters or regulate gene expression but, rather, potentiates the direct regulation of immunity-related genes by CaWRKY40, with Cys249 being a critical component of the interaction between CaWRKY28 and CaWRKY40.

\section{MATERIALS AND METHODS}

Plant materials and growth conditions.

The pepper inbred line HN42 and $N$. benthamiana plants were grown in sterilized soil in square plastic pots $(7$ by $7 \mathrm{~cm})$ in a growth room at $25^{\circ} \mathrm{C}$ and $60 \%$ humidity, with a light intensity of 60 to $70 \mu \mathrm{mol}$ photons $\mathrm{m}^{-2} \mathrm{~s}^{-1}$ and a photoperiod of 16 $\mathrm{h}$ of light and $8 \mathrm{~h}$ of darkness.

\section{Vector construction.}

We cloned the full-length open reading frames (ORFs) of CaWRKY28 and CaWRKY4O into the entry vector pDONR207 by $\mathrm{BP}$ reaction. After confirmation by sequencing, we recombined the ORFs into the destination vectors pEarleyGate103 (for C-terminal GFP fusions), pDEST-15 (for N-terminal GST fusions), or pEarleyGate201/202 (for overexpression) by LR reaction, using Gateway cloning techniques (Invitrogen, Carlsbad, CA, U.S.A.). To construct vectors for VIGS-mediated gene silencing, we PCR amplified a specific 300- to 400-bp fragment in the 3'UTRs of CaWRKY28 or CaWRKY40, whose sequence 
specificity was confirmed by Basic Local Alignment Tool (BLAST) search against the pepper genome, with specific primer pairs, using genomic DNA from pepper accession Zunla-1 as template. There are numerous CaWRKY members but they have great differences in $3^{\prime}$ UTR fragments. In order to ensure the specificity of silencing, we chose to design primers in 3'UTR. We cloned the PCR products into pDONR207 by BP reaction and confirmed the identity of the clones by sequencing before further subcloning into the PYL279 (pTRV2) vector, resulting in pTRV2:CaWRKY28, or pTRV2:CaWRKY40. All vectors were introduced into A. tumefaciens strain GV3101.

\section{VIGS.}

A. tumefaciens strain GV3101 cells harboring pTRV1, pTRV2:00, pTRV2:CAPHYTOENE DESATURASE (PDS), pTRV2:CaWRKY28, or pTRV2:CaWRKY40 were grown overnight in Luria-Bertani (LB) medium supplemented with appropriate antibiotics, then pelleted and resuspended to a final cell density of optical density at $600 \mathrm{~nm}\left(\mathrm{OD}_{600}\right)=0.8$ in infiltrationmedium $(10 \mathrm{mM}$ morpholineethanesulfonic acid, $10 \mathrm{mM}$ $\mathrm{MgCl}_{2}$, and $200 \mu \mathrm{M}$ acetosyringone; $\mathrm{pH}$ 5.4). A. tumefaciens strain GV3101 cells harboring pTRV1 were mixed with the cells containing pTRV2:00, pTRV2:CaPDS, pTRV2:CaWRKY28, or pTRV2:CaWRKY4O in a 1:1 ratio and infiltrated into the cotyledons of 2-week-old pepper seedlings. The plants were placed in a growth chamber at $16^{\circ} \mathrm{C}$ in the dark for $56 \mathrm{~h}$, then transferred to a growth room at $25^{\circ} \mathrm{C}$ and $60 \%$ humidity, with a light intensity of 60 to $70 \mu \mathrm{mol}$ photons $\mathrm{m}^{-2} \mathrm{~s}^{-1}$ and a photoperiod of $16 \mathrm{~h}$ of light and $8 \mathrm{~h}$ of darkness, until the plants infiltrated with pTRV2:CaPDS exhibited a bleached phenotype, indicative of successful silencing of the $P D S$ gene.

\section{Transient overexpression of CaWRKY28-GFP or CaWRKY40-GFP in pepper leaves.}

For transient overexpression analysis, A. tumefaciens strain GV3101 cells harboring the 35S:CaWRKY28-GFP or 35S: CaWRKY40-GFP constructs (and 35S:GFP as control) were grown overnight in LB medium with appropriate antibiotics, then pelleted and resuspended in infiltration medium to a final cell density corresponding to $\mathrm{OD}_{600}=0.8$. We infiltrated approximately $100 \mu \mathrm{l}$ of the cell suspension per infiltration site in the leaves of pepper plants at the eight-leaf stage using a syringe. After infiltration, we brought the plants to a growth room under the same conditions described above, until harvesting of the infiltrated leaves at the indicated time points for further use.

\section{R. solanacearum inoculation.}

Plants were inoculated with $R$. solanacearum strain FJC100301 as described previously (Qiu et al. 2018; Shen et al. 2016a). $R$. solanacearum was grown in sucrose peptone agar liquid medium at $28^{\circ} \mathrm{C}$. Cells were then pelleted and resuspended in a $10 \mathrm{mM} \mathrm{MgCl}_{2}$ solution to a final concentration of $10^{8} \mathrm{CFU} / \mathrm{ml}$, and used to inoculate pepper plants via root irrigation.

\section{Subcellular localization and BiFC assay.}

We determined the subcellular localization of CaWRKY28 as described previously (Shen et al. 2016a). A. tumefaciens strain GV3101 cells harboring the 35S:CaWRKY28-GFP construct were infiltrated into $N$. benthamiana leaves; and the GFP signal was detected $48 \mathrm{hpi}$. BiFC assays were performed as described previously (Choi et al. 2012) and the fluorescent signal in the cells of the Agrobacterium-infiltrated leaves was observed at 48 hpi. GFP fluorescence was collected on a confocal microscope.

Prokaryotic expression of fusion proteins in $\boldsymbol{E}$. coli.

To purify soluble CaWRKY28-GST or CaWRKY40-GST protein, we introduced a pDEST-17 plasmid harboring the full-length ORFs of CaWRKY28 or CaWRKY4O into E. coli strain BL21 (DE3). Expression of the fusion proteins was induced by the addition of $1 \mathrm{mM}$ (final concentration) isopropyl- $\beta$-D-thiogalactoside (IPTG) at $20^{\circ} \mathrm{C}$ for $12 \mathrm{~h}$. We confirmed the presence of the soluble proteins in the supernatant of the $E$. coli strain BL21 (DE3) cell lysate by SDS-PAGE.

\section{Pull-down assays.}

We undertook a pull-down approach to identify CaWRKY40 interactors: E. coli BL21 (DE3) cells containing pDEST-15CaWKY40 were cultivated on $\mathrm{LB}$ media to $\mathrm{OD}_{600}=0.5$ and then a solution of IPTG $(50 \mathrm{mg} / \mathrm{ml})$ was added to induce the biosynthesis of CaWRKY40-GST overnight. Next, the total protein was isolated and mixed with that isolated from $R$. solanacearum-inoculated pepper leaves (with uninfected pepper leaves as the control). Leaves were lysed in extraction buffer $(50 \mathrm{mM}$ phosphate buffer [pH 7.0], 10 mM EDTA, 0.1\% Triton X-100, 0.1\% sodium lauryl sarcosine, and $10 \mathrm{mM} \beta$-mercaptoethanol) by freezing with liquid nitrogen, and ground using a pestle and mortar. We immobilized the soluble fusion protein CaWRKY40-GST onto BeaverBeads GSH (Smart-Lifesciences) and incubated the mixture with rotation for $3 \mathrm{~h}$ at $4^{\circ} \mathrm{C}$. Proteins bound to the beads were subsequently washed with buffer A $(140 \mathrm{mM} \mathrm{NaCl}, 2.7 \mathrm{mM} \mathrm{KCl}$, $10 \mathrm{mM} \mathrm{Na}_{2} \mathrm{HPO}_{4}$, and $1.8 \mathrm{mM} \mathrm{KH}_{2} \mathrm{PO}_{4} ; \mathrm{pH} 7.4$ ) and eluted with elution buffer $\mathrm{B}(50 \mathrm{mM}$ Tris- $\mathrm{HCl}$ and $10 \mathrm{mM}$ reduced glutathione). Eluted proteins were resolved by SDS-PAGE; one swimming lane for RSI treatment and one lane for the control on the gel were cut off for LC-MS/MS analysis.

\section{LC-MS/MS analysis.}

Isolated proteins and potential CaWRKY40 interactors were analyzed on a LTQ-Orbitrap XL mass spectrometer (Thermo Fisher Scientific), as previously described (Wang et al. 2018; Zuo et al. 2001). The samples were dissolved in $10 \mu \mathrm{l}$ of a $10 \%$ formic acid solution, then analyzed using LC-MS/MS with an online sodium spray ion source. Peptide samples $(5 \mu \mathrm{l})$ were loaded into the trap column (Acclaim PepMapC18, $100 \mu \mathrm{m}$ by $2 \mathrm{~cm}$; Thermo Fisher Scientific) at a flow rate of $10 \mu \mathrm{l} / \mathrm{min}$, and subsequently separated on a 60-min gradient on the analytical column (Acclaim PepMapC18, $75 \mu \mathrm{m}$ by $15 \mathrm{~cm}$ ). The column flow was controlled at $300 \mathrm{nl} / \mathrm{min}$, and the electrospray voltage was $2 \mathrm{kV}$. Full scan spectra $(\mathrm{m} / \mathrm{z} 350$ to 1,550$)$ were obtained at a mass resolution of $60 \mathrm{~K}$, and higher energy collisional dissociation MS/MS scans were subsequently performed at a resolution of $30 \mathrm{~K}$ with a dynamic exclusion for $30 \mathrm{~s}$.

The original MS collection files were imported into Proteome Discover 2.1 for retrieval. We searched peptide fragments against the Zunla pepper database, and used BLAST searches at the NCBI database to annotate the functions of the corresponding proteins.

\section{ChIP analysis.}

We carried out ChIP assays as previously described (Qiu et al. 2018; Sun et al. 2015). Briefly, pepper leaves infiltrated with A. tumefaciens strain GV3101 cells harboring 35S:00, 35S:CaWRKY28-GFP, or 35S:CaWRKY40-GFP constructs were collected at 48 hpi for chromatin isolation. Extracted chromatins were sonicated to generate DNA fragments between 200 and $500 \mathrm{bp}$ in length, which we incubated with magnetic beads (Thermo Fisher Scientific) linked to anti-GFP antibody for $2 \mathrm{~h}$ at $4^{\circ} \mathrm{C}$, following the manufacturer's instructions. The immunoprecipitated DNA fragments were used as template for semiquantitative PCR or RT-qPCR with the specific primers listed in Supplementary Table S1.

\section{MST assay.}

We assessed the interaction between CaWRKY28(m) and CaWRKY40 by MST assay, as previously described (Harazi 
et al. 2017). Briefly, GFP (as a control) or CaWRKY28-GFP was used as a fluorescent label, while CaWRKY40-GST purified from E. coli strain BL21 (DE3) was used as a nonfluorescent label. Protein interaction between CaWRKY28-GFP or GFP and CaWRKY40-GST was measured at various CaWRKY40-GST concentrations with $20 \mathrm{nM}$ CaWRKY28(m)-GFP or GFP. We diluted the CaWRKY40-GST solution to a range of concentrations from $1.0 \mathrm{E}^{-10}$ to $1.0 \mathrm{E}^{-3} \mathrm{nM}$, then incubated the CaWRKY40-GST fusion protein with $20 \mathrm{nM}$ labeled protein for $10 \mathrm{~min}$ in interaction buffer. The samples were then loaded into Monolith NT.115 Capillaries (catalog MO-K002; NanoTemper Technologies) using $50 \%$ IR laser power and an LED excitation source, with $\lambda=470 \mathrm{~nm}$ at ambient temperature. We calculated the $\mathrm{Kd}$ values for CaWRKY28-GFP and CaWRKY40-GST or GFP and CaWRKY40-GST interactions using the NanoTemper Analysis 1.2.20 software (Papageorgiou et al. 2016; Zillner et al. 2012).

\section{RT-qPCR assay.}

For the RT-qPCR assay, we used a Bio-Rad Real-Time PCR system (Bio-Rad Laboratories) and the SYBR Premix Ex Taq II system (TaKaRa Bio) with specific primers listed in Supplementary Table S1. We normalized transcript levels to the reference gene CaACTIN (GQ339766). The Livak method was used to analyze the data (Livak and Schmittgen 2001).

\section{EMSA analysis.}

EMSA assays were carried out as described previously (Shen et al. 2020). To test binding against recombinant CaWRKY28 and CaWRKY28m protein, we synthesized wild-type and mutated probes by PCR, where one primer was labeled with the fluorescent dye Cy5. The recombinant proteins were incubated with Cy5-labeled wild-type or mutated probes, in $5 \times$ binding buffer. The mixture was separated by PAGE and scanned on an Odyssey CLX Infrared imager (LI-COR).

\section{Measurement of conductivity.}

We measured leaf ion leakage by determining conductivity following a previously described method (Choi et al. 2012; Hwang and Hwang 2011; Shen et al. 2016a). Leaf disks (6 mm in diameter) were taken from pepper leaves infiltrated with A. tumefaciens strain cells harboring an empty vector, 35S:CaWRKY28-GFP, 35S:CaWRKY40-GFP, or the 1:1 mixture of A. tumefaciens strain cells containing 35S:CaWRKY28GFP and 35S:CaWRKY28-GFP. The disks were harvested at the indicated time points, then incubated in $5 \mathrm{ml}$ of doubledistilled water for $1 \mathrm{~h}$ at $28^{\circ} \mathrm{C}$. Ion conductivity was measured using a Mettler Toledo 326 ion meter (Mettler Toledo).

\section{Statistical analyses.}

Statistical analysis in the present study was performed with the DPS software package. Data represented the means \pm standard deviation obtained from three or four replicates; different uppercase letters above the bars indicated significant differences among means $(P<0.01)$, as calculated with Fisher's protected least significant difference test.

\section{AUTHOR-RECOMMENDED INTERNET RESOURCES}

China National GeneBank: https://db.cngb.org/search/?q=CNP0001104 Pepper genome platform (PGP): http://peppergenome.snu.ac.kr

\section{ACKNOWLEDGMENTS}

We thank M. D. Curtis for kindly providing the Gateway destination vectors and S. P. Dinesh-Kumar of Yale University for the pTRV1 and pTRV2 vectors.

\section{LITERATURE CITED}

Abeysinghe, J. K., Lam, K. M., and Ng, D. W. 2019. Differential regulation and interaction of homoeologous WRKY18 and WRKY40 in Arabidopsis allotetraploids and biotic stress responses. Plant J. 97:352-367.

Alves, M. S., Dadalto, S. P., Gonçalves, A. B., de Souza, G. B., Barros, V. A., and Fietto, L. G. 2014. Transcription factor functional proteinprotein interactions in plant defense responses. Proteomes 2:85-106.

Amorim, L. L. B., da Fonseca Dos Santos, R., Neto, J. P. B., GuidaSantos, M., Crovella, S., and Benko-Iseppon, A. M. 2017. Transcription factors involved in plant resistance to pathogens. Curr. Protein Pept. Sci. 18:335-351.

Birkenbihl, R. P., Kracher, B., Roccaro, M., and Somssich, I. E. 2017a. Induced genome-wide binding of three Arabidopsis WRKY transcription factors during early MAMP-triggered immunity. Plant Cell 29:20-38.

Birkenbihl, R. P., Liu, S., and Somssich, I. E. 2017b. Transcriptional events defining plant immune responses. Curr. Opin. Plant Biol. 38:1-9.

Boller, T., and Felix, G. 2009. A renaissance of elicitors: Perception of microbe-associated molecular patterns and danger signals by patternrecognition receptors. Annu. Rev. Plant Biol. 60:379-406.

Cai, H., Yang, S., Yan, Y., Xiao, Z., Cheng, J., Wu, J., Qiu, A., Lai, Y., Mou, S., Guan, D., Huang, R., and He, S. 2015. CaWRKY6 transcriptionally activates CaWRKY40, regulates Ralstonia solanacearum resistance, and confers high-temperature and high-humidity tolerance in pepper. J. Exp. Bot. 66:3163-3174.

Chakraborty, J., Ghosh, P., Sen, S., and Das, S. 2018. Epigenetic and transcriptional control of chickpea WRKY40 promoter activity under Fusarium stress and its heterologous expression in Arabidopsis leads to enhanced resistance against bacterial pathogen. Plant Sci. 276:250-267.

Chen, Y. Y., Lin, Y. M., Chao, T. C., Wang, J. F., Liu, A. C., Ho, F. I., and Cheng, C. P. 2009. Virus-induced gene silencing reveals the involvement of ethylene-, salicylic acid- and mitogen-activated protein kinase-related defense pathways in the resistance of tomato to bacterial wilt. Physiol. Plant. 136:324-335.

Chi, Y., Yang, Y., Zhou, Y., Zhou, J., Fan, B., Yu, J. Q., and Chen, Z. 2013. Protein-protein interactions in the regulation of WRKY transcription factors. Mol. Plant 6:287-300.

Choi, D. S., and Hwang, B. K. 2011. Proteomics and functional analyses of pepper abscisic acid-responsive 1 (ABR1), which is involved in cell death and defense signaling. Plant Cell 23:823-842.

Choi, D. S., Hwang, I. S., and Hwang, B. K. 2012. Requirement of the cytosolic interaction between PATHOGENESIS-RELATED PROTEIN10 and LEUCINE-RICH REPEAT PROTEIN1 for cell death and defense signaling in pepper. Plant Cell 24:1675-1690.

Choi, D. S., Kim, N. H., and Hwang, B. K. 2015. The pepper phosphoenolpyruvate carboxykinase CaPEPCK1 is involved in plant immunity against bacterial and oomycete pathogens. Plant Mol. Biol. 89:99-111.

Choi, H. W., Lee, B. G., Kim, N. H., Park, Y., Lim, C. W., Song, H. K., and Hwang, B. K. 2008. A role for a menthone reductase in resistance against microbial pathogens in plants. Plant Physiol. 148:383-401.

Ciolkowski, I., Wanke, D., Birkenbihl, R. P., and Somssich, I. E. 2008. Studies on DNA-binding selectivity of WRKY transcription factors lend structural clues into WRKY-domain function. Plant Mol. Biol. 68:81-92.

Cui, H., Tsuda, K., and Parker, J. E. 2015. Effector-triggered immunity: From pathogen perception to robust defense. Annu. Rev. Plant Biol 66:487-511.

Dang, F., Wang, Y., She, J., Lei, Y., Liu, Z., Eulgem, T., Lai, Y., Lin, J., Yu, L., Lei, D., Guan, D., Li, X., Yuan, Q., and He, S. 2014. Overexpression of CaWRKY27, a subgroup IIe WRKY transcription factor of Capsicum annuиm, positively regulates tobacco resistance to Ralstonia solanacearum infection. Physiol. Plant. 150:397-411.

Dang, F. F., Wang, Y. N., Yu, L., Eulgem, T., Lai, Y., Liu, Z. Q., Wang, X., Qiu, A. L., Zhang, T. X., Lin, J., Chen, Y. S., Guan, D. Y., Cai, H. Y., Mou, S. L., and He, S. L. 2013. CaWRKY40, a WRKY protein of pepper, plays an important role in the regulation of tolerance to heat stress and resistance to Ralstonia solanacearum infection. Plant Cell Environ. 36:757-774.

Dong, J., Chen, C., and Chen, Z. 2003. Expression profiles of the Arabidopsis WRKY gene superfamily during plant defense response. Plant Mol. Biol. 51:21-37.

Eulgem, T., Rushton, P. J., Robatzek, S., and Somssich, I. E. 2000. The WRKY superfamily of plant transcription factors. Trends Plant Sci. 5:199-206.

Eulgem, T., and Somssich, I. E. 2007. Networks of WRKY transcription factors in defense signaling. Curr. Opin. Plant Biol. 10:366-371.

Gebhardt, C. 2016. The historical role of species from the Solanaceae plant family in genetic research. Theor. Appl. Genet. 129:2281-2294. 
Grunewald, W., De Smet, I., De Rybel, B., Robert, H. S., van de Cotte, B., Willemsen, V., Gheysen, G., Weijers, D., Friml, J., and Beeckman, T. 2013. Tightly controlled WRKY23 expression mediates Arabidopsis embryo development. EMBO Rep. 14:1136-1142.

Harazi, A., Becker-Cohen, M., Zer, H., Moshel, O., Hinderlich, S., and Mitrani-Rosenbaum, S. 2017. The interaction of UDP- $N$-acetylglucosamine 2-epimerase/ $N$-acetylmannosamine kinase (GNE) and alphaactinin 2 is altered in GNE myopathy M743T mutant. Mol. Neurobiol. 54:2928-2938.

Hussain, A., Li, X., Weng, Y., Liu, Z., Ashraf, M. F., Noman, A., Yang, S., Ifnan, M., Qiu, S., Yang, Y., Guan, D., and He, S. 2018. CaWRKY22 acts as a positive regulator in pepper response to Ralstonia solanacearum by constituting networks with CaWRKY6, CaWRKY27, CaWRKY40, and CaWRKY58. Int. J. Mol. Sci. 19:1426.

Hwang, I. S., and Hwang, B. K. 2011. The pepper mannose-binding lectin gene CaMBL1 is required to regulate cell death and defense responses to microbial pathogens. Plant Physiol. 155:447-463.

Ifnan Khan, M., Zhang, Y., Liu, Z., Hu, J., Liu, C., Yang, S., Hussain, A., Furqan Ashraf, M., Noman, A., Shen, L., Xia, X., Yang, F., Guan, D., and He, S. 2018. CaWRKY4Ob in Pepper Acts as a Negative Regulator in Response to Ralstonia solanacearum by Directly Modulating Defense Genes Including CaWRKY40. Int. J. Mol. Sci. 19:1403.

Kanofsky, K., Bahlmann, A. K., Hehl, R., and Dong, D. X. 2017. Combinatorial requirement of $\mathrm{W}$ - and WT-boxes in microbe-associated molecular pattern-responsive synthetic promoters. Plant Cell Rep. 36:971-986.

Kim, N. H., Lee, D. H., Choi, D. S., and Hwang, B. K. 2015. The pepper GNA-related lectin and PAN domain protein gene, $C a G L P 1$, is required for plant cell death and defense signaling during bacterial infection. Plant Sci. 241:307-315.

Lebeau, A., Daunay, M. C., Frary, A., Palloix, A., Wang, J. F., Dintinger, J., Chiroleu, F., Wicker, E., and Prior, P. 2011. Bacterial wilt resistance in tomato, pepper, and eggplant: Genetic resources respond to diverse strains in the Ralstonia solanacearum species complex. Phytopathology 101:154-165.

Le Roux, C., Huet, G., Jauneau, A., Camborde, L., Trémousaygue, D., Kraut, A., Zhou, B., Levaillant, M., Adachi, H., Yoshioka, H., Raffaele, S., Berthomé, R., Couté, Y., Parker, J. E., and Deslandes, L. 2015. A receptor pair with an integrated decoy converts pathogen disabling of transcription factors to immunity. Cell 161:1074-1088.

Liu, S., Kracher, B., Ziegler, J., Birkenbihl, R. P., and Somssich, I. E. 2015. Negative regulation of ABA signaling by WRKY33 is critical for Arabidopsis immunity towards Botrytis cinerea 2100. eLife 4:e07295.

Liu, Z.-Q., Shi, L.-P., Yang, S., Qiu, S.-S., Ma, X.-L., Cai, J.-S., Guan, D.-Y., Wang, Z.-H., and He, S.-L. 2020. A conserved double-W box in the promoter of CaWRKY40 mediates autoregulation during response to pathogen attack and heat stress in pepper. Mol. Plant Pathol. 22:3-18.

Liu, Z. Q., Yan, L., Wu, Z., Mei, C., Lu, K., Yu, Y. T., Liang, S., Zhang, X. F., Wang, X. F., and Zhang, D. P. 2012. Cooperation of three WRKYdomain transcription factors WRKY18, WRKY40, and WRKY60 in repressing two ABA-responsive genes $A B I 4$ and $A B I 5$ in Arabidopsis. J. Exp. Bot. 63:6371-6392.

Livak, K. J., and Schmittgen, T. D. 2001. Analysis of relative gene expression data using real-time quantitative PCR and the $2^{-\triangle \Delta C T}$ method. Methods 25:402-408.

Machens, F., Becker, M., Umrath, F., and Hehl, R. 2014. Identification of a novel type of WRKY transcription factor binding site in elicitorresponsive cis-sequences from Arabidopsis thaliana. Plant Mol. Biol. 84:371-385

Mamphogoro, T. P., Babalola, O. O., and Aiyegoro, O. A. 2020. Sustainable management strategies for bacterial wilt of sweet peppers (Capsicum anпuиm) and other Solanaceous crops. J. Appl. Microbiol. 129:496-508.

Nuruzzaman, M., Sharoni, A. M., and Kikuchi, S. 2013. Roles of NAC transcription factors in the regulation of biotic and abiotic stress responses in plants. Front. Microbiol. 4:248.

Pan, I. C., Li, C. W., Su, R. C., Cheng, C. P., Lin, C. S., and Chan, M. T. 2010. Ectopic expression of an EAR motif deletion mutant of SIERF3 enhances tolerance to salt stress and Ralstonia solanacearum in tomato. Planta 232:1075-1086.

Pandey, S. P., Roccaro, M., Schön, M., Logemann, E., and Somssich, I. E. 2010. Transcriptional reprogramming regulated by WRKY18 and WRKY40 facilitates powdery mildew infection of Arabidopsis. Plant J. 64:912-923.
Pandey, S. P., and Somssich, I. E. 2009. The role of WRKY transcription factors in plant immunity. Plant Physiol. 150:1648-1655.

Papageorgiou, A. C., Adam, P. S., Stavros, P., Nounesis, G., Meijers, R., Petratos, K., and Vorgias, C. E. 2016. HU histone-like DNA-binding protein from Thermus thermophilus: Structural and evolutionary analyses. Extremophiles 20:695-709.

Pennington, H. G., Gheorghe, D. M., Damerum, A., Pliego, C., Spanu, P. D., Cramer, R., and Bindschedler, L. V. 2016. Interactions between the powdery mildew effector BEC1054 and barley proteins identify candidate host targets. J. Proteome Res. 15:826-839.

Qiu, A., Lei, Y., Yang, S., Wu, J., Li, J., Bao, B., Cai, Y., Wang, S., Lin, J., Wang, Y., Shen, L., Cai, J., Guan, D., and He, S. 2018. CaC3H14 encoding a tandem $\mathrm{CCCH}$ zinc finger protein is directly targeted by CaWRKY40 and positively regulates the response of pepper to inoculation by Ralstonia solanacearum. Mol. Plant Pathol. 19:2221-2235.

Rensink, W. A., Lee, Y., Liu, J., Iobst, S., Ouyang, S., and Buell, C. R. 2005. Comparative analyses of six solanaceous transcriptomes reveal a high degree of sequence conservation and species-specific transcripts. BMC Genomics 6:124.

Rushton, P. J., Somssich, I. E., Ringler, P., and Shen, Q. J. 2010. WRKY transcription factors. Trends Plant Sci. 15:247-258.

Sarris, P. F., Duxbury, Z., Huh, S. U., Ma, Y., Segonzac, C., Sklenar, J., Derbyshire, P., Cevik, V., Rallapalli, G., Saucet, S. B., Wirthmueller, L., Menke, F. L. H., Sohn, K. H., and Jones, J. D. G. 2015. A plant immune receptor detects pathogen effectors that target WRKY transcription factors. Cell 161:1089-1100.

Segonzac, C., Newman, T. E., Choi, S., Jayaraman, J., Choi, D. S., Jung, G. Y., Cho, H., Lee, Y. K., and Sohn, K. H. 2017. A conserved EAR motif is required for avirulence and stability of the Ralstonia solanacearum effector PopP2 in planta. Front. Plant Sci. 8:1330.

Shen, L., Liu, Z., Yang, S., Yang, T., Liang, J., Wen, J., Liu, Y., Li, J., Shi, L., Tang, Q., Shi, W., Hu, J., Liu, C., Zhang, Y., Lin, W., Wang, R., Yu, H., Mou, S., Hussain, A., Cheng, W., Cai, H., He, L., Guan, D., Wu, Y., and He, S. 2016a. Pepper CabZIP63 acts as a positive regulator during Ralstonia solanacearum or high temperature-high humidity challenge in a positive feedback loop with CaWRKY40. J. Exp. Bot. 67:2439-2451.

Shen, L., Yang, S., Yang, F., Guan, D., and He, S. 2020. CaCBL1 acts as a positive regulator in pepper response to Ralstonia solanacearum. Mol. Plant-Microbe Interact 33:945-957.

Shen, L., Yang, S., Yang, T., Liang, J., Cheng, W., Wen, J., Liu, Y., Li, J., Shi, L., Tang, Q., Shi, W., Hu, J., Liu, C., Zhang, Y., Mou, S., Liu, Z., Cai, H., He, L., Guan, D., Wu, Y., and He, S. 2016b. CaCDPK15 positively regulates pepper responses to Ralstonia solanacearum inoculation and forms a positive-feedback loop with CaWRKY40 to amplify defense signaling. Sci. Rep. 6:22439.

Sun, T., Zhang, Y., Li, Y., Zhang, Q., Ding, Y., and Zhang, Y. 2015. ChIPseq reveals broad roles of SARD1 and $\mathrm{CBP} 60 \mathrm{~g}$ in regulating plant immunity. Nat. Commun. 6:10159.

Takada, S. 2013. Post-embryonic induction of ATML1-SRDX alters the morphology of seedlings. PLoS One 8:e79312.

Thera, A. T., Jacobsen, B. J., and Neher, O. T. 2010. Bacterial wilt of Solanaceae caused by Ralstonia solanacearum race 1 biovar 3 in Mali. Plant Dis. 94:372.

Tsuda, K., and Katagiri, F. 2010. Comparing signaling mechanisms engaged in pattern-triggered and effector-triggered immunity. Curr. Opin. Plant Biol. 13:459-465.

Tsuda, K., Sato, M., Stoddard, T., Glazebrook, J., and Katagiri, F. 2009. Network properties of robust immunity in plants. PLoS Genet. 5:e1000772.

Tsuda, K., and Somssich, I. E. 2015. Transcriptional networks in plant immunity. New Phytol. 206:932-947.

Wang, P., Zhao, Y., Li, Z., Hsu, C. C., Liu, X., Fu, L., Hou, Y. J., Du, Y., Xie, S., Zhang, C., Gao, J., Cao, M., Huang, X., Zhu, Y., Tang, K., Wang, X., Tao, W. A., Xiong, Y., and Zhu, J. K. 2018. Reciprocal regulation of the TOR kinase and $\mathrm{ABA}$ receptor balances plant growth and stress response. Mol. Cell 69:100-112.e6.

Wang, Y., Dang, F., Liu, Z., Wang, X., Eulgem, T., Lai, Y., Yu, L., She, J., Shi, Y., Lin, J., Chen, C., Guan, D., Qiu, A., and He, S. 2013. CaWRKY58, encoding a group I WRKY transcription factor of Capsicum annuиm, negatively regulates resistance to Ralstonia solanacearum infection. Mol. Plant Pathol. 14:131-144.

Wu, F., and Tanksley, S. D. 2010. Chromosomal evolution in the plant family Solanaceae. BMC Genomics 11:182

Xu, X., Chen, C., Fan, B., and Chen, Z. 2006. Physical and functional interactions between pathogen-induced Arabidopsis WRKY18, WRKY40, and WRKY60 transcription factors. Plant Cell 18:1310-1326. 
Zhang, H.-X., Ali, M., Feng, X.-H., Jin, J.-H., Huang, L.-J., Khan, A., Lv, J.-G., Gao, S.-Y., Luo, D.-X., and Gong, Z.-H. 2019. A novel transcription factor CaSBP12A gene negatively regulates the defense response against Phytophthora capsici in pepper (Capsicum annuum L.). Int. J. Mol. Sci. 20:48.

Zhang, L., Li, Y., Lu, W., Meng, F., Wu, C. A., and Guo, X. 2012. Cotton GhMKK5 affects disease resistance, induces HR-like cell death, and reduces the tolerance to salt and drought stress in transgenic Nicotiana benthamiana. J. Exp. Bot. 63:3935-3951.

Zhang, Y., and Wang, L. 2005. The WRKY transcription factor superfamily: Its origin in eukaryotes and expansion in plants. BMC Evol. Biol. 5:1.
Zillner, K., Jerabek-Willemsen, M., Duhr, S., Braun, D., Längst, G., and Baaske, P. 2012. Microscale thermophoresis as a sensitive method to quantify protein: Nucleic acid interactions in solution. Pages 241-252 in: Functional Genomics. Methods in Molecular Biology (Methods and Protocols), vol. 815. M. Kaufmann and C. Klinger, eds. Springer, New York, NY, U.S.A.

Zuo, X., Echan, L., Hembach, P., Tang, H. Y., Speicher, K. D., Santoli, D., and Speicher, D. W. 2001. Towards global analysis of mammalian proteomes using sample prefractionation prior to narrow $\mathrm{pH}$ range two-dimensional gels and using one-dimensional gels for insoluble and large proteins. Electrophoresis 22:1603-1615. 